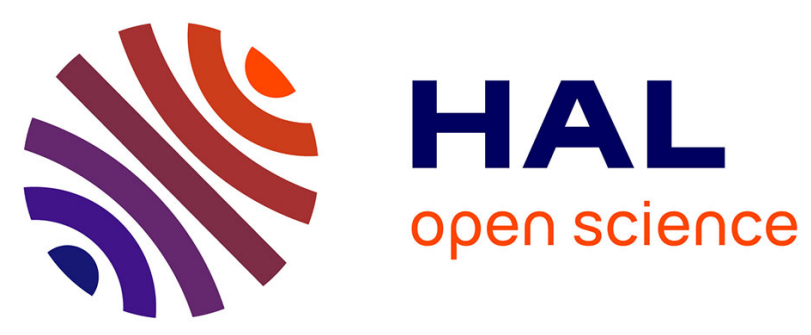

\title{
Dynamical interactions between the coherent motion and small scales in a cylinder wake
}

\author{
F. Thiesset, L. Danaila, R. a. Antonia
}

\section{To cite this version:}

F. Thiesset, L. Danaila, R. a. Antonia. Dynamical interactions between the coherent motion and small scales in a cylinder wake. Journal of Fluid Mechanics, 2014, 749, pp.201 - 226. 10.1017/jfm.2014.222 . hal-01660263

\section{HAL Id: hal-01660263 \\ https://hal.science/hal-01660263}

Submitted on 28 Mar 2019

HAL is a multi-disciplinary open access archive for the deposit and dissemination of scientific research documents, whether they are published or not. The documents may come from teaching and research institutions in France or abroad, or from public or private research centers.
L'archive ouverte pluridisciplinaire HAL, est destinée au dépôt et à la diffusion de documents scientifiques de niveau recherche, publiés ou non, émanant des établissements d'enseignement et de recherche français ou étrangers, des laboratoires publics ou privés. 


\title{
Dynamical interactions between the coherent motion and small scales in a cylinder wake
}

\author{
F. THIES S E T ${ }^{1,2}$, L. D A N A I L A ${ }^{1} \dagger$, \\ AND R. A. A N T ONIA ${ }^{2}$ \\ ${ }^{1}$ CORIA, Avenue de l'Université, BP 12, 76801 Saint Etienne du Rouvray, France \\ ${ }^{2}$ School of Engineering, University of Newcastle, NSW 2308, Australia
}

(Received 28 March 2019)

Most turbulent flows are characterized by coherent motion (CM), whose dynamics reflect the initial and boundary conditions of the flow and are more predictable than that of the random motion (RM). The major question we address here is the dynamical interaction between the CM and the RM, at a given scale, in a flow where the CM exhibits a strong periodicity and can therefore be readily distinguished from the RM. The question is relevant at any Reynolds number, but is of capital importance at finite Reynolds numbers, for which a clear separation between the largest and the smallest scales may not exist.

Both analytical and experimental tools are used to address this issue. First, phaseaveraged structure functions are defined and further used to condition the RM kinetic energy at a scale $r$ on the phase $\phi$ of the CM. This tool allows the dependence of the $\mathrm{RM}$ to be followed as a function of the CM dynamics. Scale-by-scale energy budget equations are established on the basis of phase-averaged structure functions. They reveal that energy transfer at a scale $r$ is sensitive to an additional forcing mechanism due to the CM. Second, these concepts are tested using hot-wire measurements in a cylinder wake, in which the CM is characterized by a well-defined periodicity. Because the interaction between large and small scales is most likely enhanced at moderate/low Reynolds numbers, and is also likely to depend on the amplitude of the CM, we choose to test our findings against experimental data at $R_{\lambda} \sim 10^{2}$ and for downstream distances in the range $10 \leq x / D \leq 40$. The effects of an increasing Reynolds number are also discussed.

It is shown that: (i) a simple analytical expression describes the second-order structure functions of the purely coherent motion. The energy of the CM is not associated with any single scale; instead, its energy is distributed over a range of scales. (ii) Close to the obstacle, the influence of the CM is perceptible even at the smallest scales, the energy of which is enhanced when the coherent strain is maximum. Further downstream from the cylinder, the CM clearly affects the largest scales, but the smallest scales are not likely to depend explicitly on the CM. (iii) The isotropic formulation of the random motion energy budget compares favorably with experimental results.

Key words: Turbulent wake, coherent motion, scale-by-scale energy budget

\section{Introduction}

Turbulent flows give rise to a wide and continuous range of scales. The largest eddies reflect the way the kinetic energy is injected in the system and therefore depend on the

† Email address for correspondence: danaila@coria.fr 
type of flow. In contrast, one frequently asserts that the anisotropic and non-universal influence of the largest scales diminishes during the first non-linear local interactions and is thus expected to decline at the smallest scales. Consequently, it is still often postulated that the smallest scales have the best prospect of being universal or quasiuniversal (Sreenivasan \& Antonia 1997) a paradigm usually attributed to Kolmogorov $(1941 a, b)$ (hereafter K41). One of the premises of K41 is that small-scale turbulence at sufficiently high Reynolds numbers is statistically independent of the large scales, and is stationary, homogeneous and isotropic. Taking these premises for granted, K41 states that $(i)$ the statistical properties of the small scales are determined universally by $\nu$ and $\bar{\epsilon}$ (the kinematic viscosity and the mean energy dissipation rate); (ii) those in the inertial range (if the Reynolds number allows one to exist) are determined by $\bar{\epsilon}$ only.

K41 was formulated for large Reynolds numbers, whereas the turbulent flows generally encountered in practical situations are characterized by low to moderate Reynolds numbers. It is essential to recall at this stage that significant departures from the scaling proposed by K41 are due to finite Reynolds number effects. Although the small scales have indeed the 'best prospect' of being universal, they may also exhibit strong departures from universality, depending both on the statistics used to filter the small scales and on the physical quantity chosen to represent the largest ones. Pragmatically speaking, testing small-scale universality (SSU) involves at least three parameters: the mathematical functions used to filter both the small scales and the large scales, as well as the Reynolds number of the flow. In this regard, the validity of SSU may depend on the severity of the chosen test. Before providing a general classification of these tests, it should be kept in mind that 'universality' implicitly supposes local isotropy. Testing SSU can be done at several levels.

- A basic level, which involves low (second-and-third) order statistics, e.g. spectra, second-and-third order structure functions. In physical space, one common way to retain small scales is considering increments at that scale, which represent motions at scales $\leq r$ (not only at the particular scale $r$ ). It can be argued that (Kholmyansky \& Tsinober 2008) neither the original derivation of the Kolmogorov 2/3 and 4/5 laws, nor all the subsequent derivations of the 4/5 law use the assumption of locality of interactions and the existence of a cascade. In obtaining the $5 / 3$ and $2 / 3$ laws, only phenomenological assumptions are necessary (K41), underpinned by the local isotropy hypothesis. Nothing is supposed about either the nature of the large scales, or the exact dynamics of the cascade, i.e. how energy flows from one scale to another scale. Nonetheless, results such as $5 / 3,2 / 3$ and $4 / 5$ (the latter is ensuing from the Navier-Stokes equations) are extremely robust and ubiquitous, provided the Reynolds number (the single parameter that dictates if SSU is verified at this level) is sufficiently high.

Danaila et al. (2004) made a a further step in understanding the role of large scales in transport equations for the second-order structure functions, thus allowing the finite Reynolds number effects to be quantified. The effect of the 'large scales' is generally represented by the processes associated with the decay, production and turbulent diffusion of the turbulent energy. The dependence of small-scale statistics on large-scale effects is in general enhanced as the Reynolds numbers decreases. This framework led Antonia \& Burattini (2006) to assess the Reynolds number that ought to be reached for K41 to be satisfied and it was proven that a Taylor-based Reynolds number as high as 50,000 is needed for decaying turbulence to reach the 4/5 law. A similar result was obtained in spectral space for Lin's equation (closed via the EDQNM approach), which includes the decay term (e.g. Tchoufag et al. (2012)). Even though analyses in spectral space can provide significant insight into the degree of the interactions between different scales in some canonical situations (Domaradzki et al. 1987), even when the flow is 
slightly inhomogeneous (e.g. Cambon \& Gréa (2013)), the strong inhomogeneity generally encountered in complex flow configurations does not allow the use of spectra and dynamical equation in Fourier space. Considerations in physical space are thus likely to be the best solution for investigating these flows. In addition, we have to mention that the analogy between the real-space and spectral-space expressions of different physical quantities is not straightforward, as the spectra strictly correspond to the energy distribution at a given wavenumber, whereas the second-order structure functions tend to represent the energy at all scales $\leq r$ (Townsend 1956; Davidson \& Pearson 2005; Mouri \& Hori 2010; Danaila et al. 2012a). Cambon et al. (2013) discussed in detail the differences and analogies between analyses in spectral and physical space. Finally, as far as we are aware, conditional statistics are not usually considered in spectral space, whereas these are often utilized in physical space.

- A refined level, for which low-order statistics representing small scales (often, increments) are conditioned by quantities representing large scales (either the mean shear, the local value of the velocity, the local phase of the coherent motion when this is pertinent, etc.). This conditioning represents a priori a more stringent test of SSU and allows for the correlations between the large scales and small scales to be highlighted.

A statistical measure of the effect of the large scales is the sum of velocities at two points separated by a distance $r$ (Hosokawa 2007; Mouri \& Hori 2010). An interesting analysis along this direction is provided by Hosokawa (2007), who demonstrated the statistical dependence of the difference and sum of velocities at two points, thus underlining the statistical dependence of small scales on the larger ones. Moreover, this author has shown that this clear dependence is fully compatible with the $4 / 5$ law. This analytical treatment was then accurately confirmed by the experiments of Kholmyansky \& Tsinober (2008) in different high Reynolds number flows. Hence, we now possess strong evidence, on both theoretical and experimental fronts, that even at very high Reynolds numbers, the way energy is distributed between different scales depends on the large scales and most likely involve both local and non-local interactions. Consequently, one should expect these interactions to be even more predominant for Reynolds numbers normally encountered in the laboratory. This issue is worth being explored and characterized analytically and hence some new statistical tools that quantify the interactions between large (and in particular the CM) and small scales has to be developed.

To this end, one possibility is to consider velocity increments, which represent motions at scales $\leq r$ (e.g. Mouri \& Hori (2010); Danaila et al. (2012a)), conditioned by another parameter characterizing motions at scales $\geq r$ (Mouri \& Hori 2010). The first study based on conditional structure functions is that of Praskovsky et al. (1993). The latter reported structure functions conditioned by the instantaneous velocity in two different high Reynolds number shear flows. These suggested a strong correlation between large and inertial subrange scales. Praskovsky et al. (1993) finally concluded that these findings are in contradiction with the random sweeping decorrelation hypothesis (Tennekes 1975) which was first stated by supposing statistical independence between eddies. Subsequently, measured structure functions were conditioned by the instantaneous velocity in the atmospheric boundary layer (Sreenivasan \& Dhruva 1998). Despite the very high Reynolds number, a strong correlation between large and small scales also emerged. According to Sreenivasan \& Dhruva (1998), this result may highlight the persisting influence of the mean shear on the smallest scales.

More recently, Blum et al. (2010, 2011) measured structure functions conditioned by the average of the velocity at points $x$ and $x+r$ in different shearless flows. These authors pointed out that small scales were perturbed by the large scales at very different levels 
depending on the flow type. They concluded that conditional structure functions provide a reference tool for comparing large-scale effects in different flows.

To summarize this overview, (i) at a basic level, considering low-order statistics of velocity increments as a small-scale filter unconditioned by large scales, leads to the SSU to be valid $(2 / 3,5 / 3,4 / 5)$, provided the Reynolds number is sufficiently high. (ii) At a refined level, if we consider small-scale statistics that are conditioned on large scales, SSU seems to break down, at least for Reynolds numbers encountered in the laboratory. This violation of SSU is not in contradiction with K41, as the latter was aimed at unconditioned statistics. Therefore, the validity of SSU clearly depends on the chosen test and the way the effect of the large scales is quantified.

A further step in understanding and quantifying the interaction between large and small scales is done in the present work. We address the issue of the dynamical dependence of low-order statistics on the large-scale activity of a Coherent Motion (CM). More precisely, rather than investigating the plausibility of SSU which is likely to be pertinent at very large Reynolds numbers, we focus here on such interactions that may occur in finite Reynolds number flows. This choice is motivated by the fact that (i) finite Reynolds flows are encountered in practical situations and (ii) these interactions are likely to be more perceptible at low Reynolds number. Investigating the nature of the interactions between CM and the small-scale motion is the principal motivation of the present work. A better understanding of the effect of the large-scale CM on the smallest scales is of major practical interest, for example for designing new efficient sub-grid scale models (O’Neil \& Meneveau 1997; Kang \& Meneveau 2002).

Because of the pioneering work of Townsend (1956) and many other researchers (e.g. Brown \& Roshko $(1974,2012)$ ), it is now well known that most shear flows contain socalled coherent structures. These are energy-containing eddies present at rather large scales, which strongly persist in time and/or in space. In terms of characteristic timescales, Thiesset et al. (2013b) showed that the life-time of the CM is much larger than that of the small scales. The topology of the organized motion depends on initial conditions and their related statistics are not universal (Antonia et al. 2002; Thiesset et al. $2013 b$ ). The plane wake flow is one possible candidate for studying the degree of SSU in presence of a CM. As a reminder, at a Reynolds number $\operatorname{Re}_{D}\left(\equiv U_{0} D / \nu, U_{0}\right.$ is the upstream velocity, $D$ the cylinder diameter and $\nu$ the kinematic viscosity) of about 45 (Williamson 1996), the well-known two-dimensional Bénard-von Kármán appears and degenerates in three dimensions at a Reynolds of about 150 (Williamson 1996). Then, for Reynolds numbers $R e_{D}>10^{3}$, the shear layers emanating from the sides of the cylinder destabilise and start being turbulent. The normalized shedding frequency, i.e. the Strouhal number, monotonically increases for a Reynolds number $R e_{D}$ between 45 and 1000 , before reaching a plateau in the range $10^{3}<R e_{D}<10^{4}$ with a value of about 0.21 (Roshko 1954). The wake flow is particularly suited for investigating the interactions between the CM and small scales for the simple reason that the CM is sharply identified in spectral space, and its frequency (or, Strouhal number) remains constant over a long distance downstream the obstacle (Roshko 1954; Williamson 1996). In other words, in contrast with some other flows as the shear layer for which the CM reveals some cycleto-cycle variations, or the boundary layer for which a large variety of coherent structures are present, the CM pertaining to the wake flow is characterized by strongly stable structures that are advected almost identically in the direction of mean flow. The information contained within the CM persists until the far field (Bisset et al. 1990; Zhou \& Antonia 1995; Thiesset et al. 2013b) and influence small-scale statistics due to non-local interactions and to a larger extent to finite Reynolds numbers effects. As far as the wake flow is concerned, it was proven that the CM can induce a strong anisotropy through the 
additional effect of the coherent strain (Thiesset et al. 2013b). It was also stated that the energy distribution and the maximum value of the energy transfer may be altered significantly depending on the amplitude of the coherent motion (Thiesset et al. 2013a). The results presented by Thiesset et al. $(2013 b, a)$ suggest that significant progress are needed to unravel the physical processes at play in the dynamical relationship between coherent and small scales.

The present study addresses a few specific issues: (i) What is the nature and degree of interaction between large and small scales? (ii) What are the energy budget equations at a given scale in flows where a CM may be discernible? (iii) Can we determine a reliable analytical expression for the distribution of energy among different scales in the presence of the CM?

To unravel these issues, the way we condition structure functions is somewhat different to that presented previously. Based on the approach advocated by Reynolds \& Hussain (1972), we propose to condition structure functions by a particular value of the phase $\phi$ arising from the phase-averaging operation.

This study focuses entirely on a circular cylinder wake flow, which is investigated by means of hot wire experiments. Different streamwise locations were studied, from $x=10 D$ to $x=40 D$ ( $D$ is the cylinder diameter) leading to a decreasing amplitude of velocity coherent fluctuations. Investigations concentrate mostly on the wake centerline, where the mean shear is absent. This allows us to focus only on the influence of the coherent motion, thus avoiding the additive effect of the mean shear.

Following the definition of the phase-conditioned structure functions ( $\$ 2.1$ ), energy budget equations for both coherent and random motion are first derived in $\S 2.2$. This allows us to highlight the relevant quantities that ought to be assessed for the interactions between the coherent motion and the small scales to be unravelled. Then, measurements in the intermediate wake of a circular cylinder are described in $\S 2.3$. One-point statistics are then presented in $\S 3.1$, with particular emphasis on the typical topology of the CM pertaining to the wake flow. The interactions between coherent and random fluctuating fields are illustrated by means of second (Subsection §3.2) and third-order (Subsection 3.3) phase-averaged structure functions. The isotropic formulation of the scale-by-scale budget of the random motion is tested against experimental data in $\S 4$. Conclusions are drawn in Section 5.

\section{Tools of investigation}

\subsection{Phase-averaged structure functions}

Before describing in detail the main achievements of the present study, we first briefly discuss the tools that allow to highlight the features of the coherent motion pertaining to the wake flow. To this end, it is first useful to separate the contribution of the CM from that of the turbulent random motion (RM). In flows where a coherent motion is discernible, one often invokes the triple decomposition (Reynolds \& Hussain 1972) according to which any fluctuating quantity $\beta$ is decomposed as

$$
\beta=\bar{\beta}+\widetilde{\beta}+\beta^{\prime}
$$

where $\bar{\beta}, \widetilde{\beta}$ and $\beta^{\prime}$ denote the mean, coherent and purely turbulent components of $\beta$ respectively. To extract the information on the coherent motion, a phase averaging is performed (hereafter noted by angular brackets), namely

$$
\langle\beta\rangle(\phi)=\bar{\beta}+\widetilde{\beta}(\phi)
$$


since by definition $\left\langle\beta^{\prime}\right\rangle=0$. $\phi$ characterizes the phase of the CM. As far as the velocity and pressure fields are concerned, the triple decomposition reads (Reynolds \& Hussain 1972)

$$
\begin{array}{rr}
U_{i}=\bar{U}_{i}+\tilde{u}_{i}+u_{i}^{\prime}, & P=\bar{P}+\tilde{p}+p^{\prime} \\
u_{i}=\tilde{u}_{i}+u_{i}^{\prime}, & p=\tilde{p}+p^{\prime}, \\
\left\langle u_{i}^{\prime}\right\rangle=0, & \left\langle p^{\prime}\right\rangle=0 .
\end{array}
$$

In the past, phase-averaging has been applied mostly to the velocity one-point statistics, in order to emphasize for example the energy contribution of the coherent motion to the Reynolds stress. However, similar treatments can be carried out concerning two-point statistics with the view of relating the temporal dynamics of the energy distribution across the scales to that of the organized motion.

Phase-averaging is thus applied to the $n-t h$ order structure functions (here, $n$ ranges from 1 to 3). Second- and third-order structure functions are as usually functions of $r$, but specific to our methodology, they are also functions of the phase $\phi$

$$
\begin{aligned}
\left\langle\left(\Delta u_{\alpha}\right)^{n}\right\rangle(\vec{r}, \phi) & =\left\langle\left[u_{\alpha}\left(\vec{X}+\frac{1}{2} \vec{r}\right)-u_{\alpha}\left(\vec{X}-\frac{1}{2} \vec{r}\right)\right]^{n}\right\rangle(\vec{r}, \phi) \\
& =\left\langle\left[u_{\alpha}(\vec{x}+\vec{r})-u_{\alpha}(\vec{x})\right]^{n}\right\rangle(\vec{r}, \phi)
\end{aligned}
$$

which represents the ensemble average of the $n-t h$ order increment $\Delta u_{\alpha}=u_{\alpha}(\vec{x}+$ $\vec{r})-u_{\alpha}(\vec{x})$ of the velocity component $u_{\alpha}$, knowing $\phi(\vec{X}) \cdot \vec{X}$ is the midpoint between two points of space $\vec{x}$ and $\vec{x}+\vec{r}$. Classical time-averaged structure functions are calculated by integrating $\left\langle\left(\Delta u_{\alpha}\right)^{n}\right\rangle$ over all values of $\phi \in[-\pi ; \pi]$ and are denoted by $\overline{\left\langle\left(\Delta u_{\alpha}\right)^{n}\right\rangle}$. An important remark is that for $n=1$,

$$
\left\langle\Delta u_{\alpha}\right\rangle=\Delta \tilde{u}_{\alpha} \neq 0 .
$$

Unlike the classical structure function, the first-order phase-averaged structure function is not zero for $r \neq 0$. However $\overline{\left\langle\Delta u_{\alpha}\right\rangle}=0$. Another result concerns the second-order structure functions of the total fluctuating velocity field $u_{\alpha}$, which is the sum of the second-order structure functions of the random and coherent motions, namely

$$
\left\langle\left(\Delta u_{\alpha}\right)^{2}\right\rangle=\left(\Delta \tilde{u}_{\alpha}\right)^{2}+\left\langle\left(\Delta u_{\alpha}^{\prime}\right)^{2}\right\rangle .
$$

The influence of the phase, on the total, organized and random kinetic energy at a given scale, can thus be assessed separately. Similar results can be obtained for third-order structure functions.

\subsection{Dynamical transport equations for phase-conditioned two-point statistics}

As a further step for investigating flows populated by CM, it is of interest to derive an energy budget equation for second-order phase-averaged structure function. The particular expected outcome is to highlight the influence of the CM dynamics on the energy distribution and thereof on the energy transferred at a scale $r$ (and smaller). One advantage of phase-averaging is that it allows transport equations to be written for both the $\mathrm{CM}$ as well as the purely RM.

\subsubsection{General formulation}

The starting point is the Navier-Stokes equation

$$
\frac{\partial U_{i}}{\partial t}+U_{j} \frac{\partial U_{i}}{\partial x_{j}}=-\frac{\partial P}{\partial x_{i}}+\nu \frac{\partial^{2} U_{i}}{\partial x_{j} \partial x_{j}},
$$


where $P$ is the kinematic pressure and $\nu$ the kinematic viscosity. The summation convention applies to repeated Roman indices. By further using Eq. (2.3), Reynolds \& Hussain (1972) obtained the dynamical equations of the coherent and random components, which are respectively

$$
\begin{gathered}
\frac{D \tilde{u}_{i}}{D t}+\tilde{u}_{j} \frac{\partial \bar{U}_{i}}{\partial x_{j}}+\frac{\partial}{\partial x_{j}}\left(\tilde{u}_{i} \tilde{u}_{j}-\overline{\tilde{u}_{i} \tilde{u}_{j}}\right)+\frac{\partial}{\partial x_{j}}\left(\left\langle u_{i}^{\prime} u_{j}^{\prime}\right\rangle-\overline{u_{i}^{\prime} u_{j}^{\prime}}\right)=-\frac{\partial \tilde{p}}{\partial x_{i}}+\nu \frac{\partial^{2} \tilde{u}_{i}}{\partial x_{j}^{2}} \\
\frac{D u_{i}^{\prime}}{D t}+\tilde{u}_{j} \frac{\partial u_{i}^{\prime}}{\partial x_{j}}+u_{j}^{\prime} \frac{\partial \bar{U}_{i}}{\partial x_{j}}+u_{j}^{\prime} \frac{\partial \tilde{u}_{i}}{\partial x_{j}}+\frac{\partial}{\partial x_{j}}\left(u_{i}^{\prime} u_{j}^{\prime}-\left\langle u_{i}^{\prime} u_{j}^{\prime}\right\rangle\right)=-\frac{\partial p^{\prime}}{\partial x_{i}}+\nu \frac{\partial^{2} u_{i}^{\prime}}{\partial x_{j}^{2}}
\end{gathered}
$$

$\frac{D}{D t}=\frac{\partial}{\partial t}+\bar{U}_{j} \frac{\partial}{\partial x_{j}}$ is the material derivative. Eqs. (2.8a) and (2.8b) are written at points $\vec{x}$ and $\vec{x}^{+}=\vec{x}+\vec{r}$ separated by a distance $\vec{r}$. Then, the equation at point $\vec{x}$ is subtracted from that at point $\vec{x}^{+}$, so that

$$
\begin{array}{r}
\frac{\partial \Delta \tilde{u}_{i}}{\partial t}+\Delta\left(\bar{U}_{j} \frac{\partial \tilde{u}_{i}}{\partial x_{j}}\right)+\Delta\left(\tilde{u}_{j} \frac{\partial \bar{U}_{i}}{\partial x_{j}}\right)+\Delta\left(\frac{\partial}{\partial x_{j}}\left(\tilde{u}_{i} \tilde{u}_{j}-\overline{\tilde{u}_{i} \tilde{u}_{j}}\right)\right) \\
+\Delta\left(\frac{\partial}{\partial x_{j}}\left(\left\langle u_{i}^{\prime} u_{j}^{\prime}\right\rangle-\overline{u_{i}^{\prime} u_{j}^{\prime}}\right)\right)=-\Delta\left(\frac{\partial \tilde{p}}{\partial x_{i}}\right)+\nu \Delta\left(\frac{\partial^{2} \tilde{u}_{i}}{\partial x_{j}^{2}}\right) \\
\frac{\partial \Delta u_{i}^{\prime}}{\partial t}+\Delta\left(\bar{U}_{j} \frac{\partial u_{i}^{\prime}}{\partial x_{j}}\right)+\Delta\left(\tilde{u}_{j} \frac{\partial u_{i}^{\prime}}{\partial x_{j}}\right)+\Delta\left(u_{j}^{\prime} \frac{\partial \bar{U}_{i}}{\partial x_{j}}\right)+\Delta\left(u_{j}^{\prime} \frac{\partial \tilde{u}_{i}}{\partial x_{j}}\right) \\
+\Delta\left(\frac{\partial}{\partial x_{j}}\left(u_{i}^{\prime} u_{j}^{\prime}-\left\langle u_{i}^{\prime} u_{j}^{\prime}\right\rangle\right)\right)=-\Delta\left(\frac{\partial p^{\prime}}{\partial x_{i}}\right)+\nu \Delta\left(\frac{\partial^{2} u_{i}^{\prime}}{\partial x_{j}^{2}}\right)
\end{array}
$$

Eq. (2.9a) is the dynamical equation of coherent velocity increments, and Eq. (2.9b) the dynamical equation of random velocity increments. We consider that the mean velocity field is sufficiently uniform for its spatial increments to be negligible compared with those of both the random and coherent motions, viz. $\Delta \overline{U_{i}} \ll \Delta \tilde{u}_{i}, \Delta \overline{U_{i}} \ll \Delta u_{i}^{\prime}$. Hence, the two following transport equations for $\Delta \tilde{u}_{i}$ and $\Delta u_{i}^{\prime}$ can be derived

$$
\begin{array}{r}
\frac{D \Delta \tilde{u}_{i}}{D t}+\Delta \tilde{u}_{j} \frac{\partial \bar{U}_{i}}{\partial x_{j}}+\Delta\left(\frac{\partial}{\partial x_{j}}\left(\tilde{u}_{i} \tilde{u}_{j}-\overline{\tilde{u}_{i} \tilde{u}_{j}}\right)\right) \\
+\Delta\left(\frac{\partial}{\partial x_{j}}\left(\left\langle u_{i}^{\prime} u_{j}^{\prime}\right\rangle-\overline{u_{i}^{\prime} u_{j}^{\prime}}\right)\right)=-\Delta\left(\frac{\partial \tilde{p}}{\partial x_{i}}\right)+\nu \Delta\left(\frac{\partial^{2} \tilde{u}_{i}}{\partial x_{j}^{2}}\right), \\
\frac{D \Delta u_{i}^{\prime}}{D t}+\Delta\left(\tilde{u}_{j} \frac{\partial u_{i}^{\prime}}{\partial x_{j}}\right)+\Delta u_{j}^{\prime} \frac{\partial \bar{U}_{i}}{\partial x_{j}}+\Delta\left(u_{j}^{\prime} \frac{\partial \tilde{u}_{i}}{\partial x_{j}}\right) \\
+\Delta\left(\frac{\partial}{\partial x_{j}}\left(u_{i}^{\prime} u_{j}^{\prime}-\left\langle u_{i}^{\prime} u_{j}^{\prime}\right\rangle\right)\right)=-\Delta\left(\frac{\partial p^{\prime}}{\partial x_{i}}\right)+\nu \Delta\left(\frac{\partial^{2} u_{i}^{\prime}}{\partial x_{j}^{2}}\right) .
\end{array}
$$

As proposed by Hill (2001), Danaila et al. (2002), Danaila et al. (2004), we use derivatives with respect to the midpoint $\vec{X}$ defined by $\vec{X}=\left(\vec{x}+\vec{x}^{+}\right) / 2$, so that $\partial / \partial x_{j}=-\partial / \partial r_{j}+$ $\partial / 2 \partial X_{j}$ and $\partial / \partial x_{j}^{+}=\partial / \partial r_{j}+\partial / 2 \partial X_{j}$. We further assume that the two points $\vec{x}$ and $\vec{x}^{+}$are independent (Hill 2001; Danaila et al. 2002, 2004), viz. $\partial u_{i} / x_{j}^{+}=\partial u_{i}^{+} / x_{j}=0$. After multiplying Eq. $(2.10 a)$ by $2 \Delta \tilde{u}_{i}$ and Eq. $(2.10 b)$ by $2 \Delta u_{i}^{\prime}$, applying phase averaging followed by time averaging, and noting that

$$
\begin{aligned}
& \left\langle\Delta u_{j}^{\prime} \Delta u_{i}^{\prime}\right\rangle=\left\langle\Delta u_{i} \Delta u_{j}\right\rangle-\Delta \tilde{u}_{i} \Delta \tilde{u}_{j} \\
& \left\langle\Delta u_{j} \Delta q^{2}\right\rangle=\Delta \tilde{u}_{j} \Delta \tilde{q}^{2}+\Delta \tilde{u}_{j}\left\langle\Delta q^{\prime 2}\right\rangle+\left\langle\Delta u_{j}^{\prime} \Delta q^{\prime 2}\right\rangle+2 \Delta \tilde{u}_{i}\left\langle\Delta u_{j}^{\prime} \Delta u_{i}^{\prime}\right\rangle,
\end{aligned}
$$


we finally obtain the scale-by-scale energy budget of the coherent motion (Thiesset et al. 2011)

$$
\begin{array}{r}
\overline{\frac{D}{D t} \Delta \tilde{q}^{2}}+\frac{\partial}{\partial X_{j}} \overline{\left[\overline{\Sigma \tilde{u}_{j} \Delta \tilde{q}^{2}}+2 \overline{\left\langle\Sigma u_{j}^{\prime} \Delta u_{i}^{\prime}\right\rangle \Delta \tilde{u}_{i}}+2 \overline{\Delta \tilde{u}_{i} \Delta \tilde{p}}\right]+2 \overline{\Delta \tilde{u}_{i} \Delta \tilde{u}_{j}} \frac{\partial \bar{U}_{i}}{\partial x_{j}}} \\
-\left\langle\overline{\left\langle u_{j}^{\prime} \Delta u_{i}^{\prime}\right\rangle \frac{\partial}{\partial X_{j}} \Delta \tilde{u}_{i}}+\frac{\partial}{\partial r_{j}} \overline{\Delta \tilde{u}_{j} \Delta \tilde{q}^{2}}+2 \Delta \tilde{u}_{i} \frac{\partial}{\partial r_{j}}\left\langle\Delta u_{i}^{\prime} \Delta u_{j}^{\prime}\right\rangle\right. \\
-\nu\left[\left(2 \frac{\partial^{2}}{\partial r_{j}^{2}}+\frac{1}{2} \frac{\partial^{2}}{\partial X_{j}^{2}}\right) \overline{\Delta \tilde{q}^{2}}+2 \Sigma\left(\overline{\frac{\partial \tilde{u}_{i}}{\partial x_{j}} \frac{\partial \tilde{u}_{j}}{\partial x_{i}}}\right)\right]=-2 \Sigma \overline{\tilde{\epsilon}}
\end{array}
$$

For the random motion, the corresponding energy budget equation is (Thiesset et al. 2011)

$$
\begin{array}{r}
\overline{\frac{D}{D t} \Delta q^{\prime 2}}+\frac{\partial}{\partial X_{j}}\left[\overline{\Sigma u_{j}^{\prime} \Delta q^{\prime 2}}+\overline{\Sigma \tilde{u}_{j}\left\langle\Delta q^{\prime 2}\right\rangle}+2 \overline{\Delta u_{i}^{\prime} \Delta p^{\prime}}\right]+2 \overline{\Delta u_{i}^{\prime} \Delta u_{j}^{\prime}} \frac{\partial \bar{U}_{i}}{\partial x_{j}} \\
+\overline{\left\langle\Sigma u_{j}^{\prime} \Delta u_{i}^{\prime}\right\rangle \frac{\partial}{\partial X_{j}} \Delta \tilde{u}_{i}}+\frac{\partial}{\partial r_{j}}\left(\overline{\left\langle\Delta u_{j} \Delta q^{2}\right\rangle}-\overline{\Delta \tilde{u}_{j} \Delta \tilde{q}^{2}}\right)-2 \Delta \tilde{u}_{i} \frac{\partial}{\partial r_{j}}\left\langle\Delta u_{i}^{\prime} \Delta u_{j}^{\prime}\right\rangle \\
-\nu\left[\left(2 \frac{\partial^{2}}{\partial r_{j}^{2}}+\frac{1}{2} \frac{\partial^{2}}{\partial X_{j}^{2}}\right) \overline{\Delta q^{\prime 2}}+2 \Sigma\left(\overline{\left.\left.\frac{\partial u_{i}^{\prime}}{\partial x_{j}} \frac{\partial u_{j}^{\prime}}{\partial x_{i}}\right)\right]=-2 \Sigma \overline{\epsilon^{\prime}}}\right.\right.
\end{array}
$$

$\overline{\Delta \tilde{q}^{2}}=\overline{\Delta \tilde{u}_{i} \Delta \tilde{u}_{i}}$ and $\overline{\Delta q^{\prime 2}}=\overline{\Delta u_{i}^{\prime} \Delta u_{i}^{\prime}}$ are the coherent and random kinetic energies at a given scale respectively. $\Sigma \bullet=\bullet(\vec{x}+\vec{r})+\bullet(\vec{x})$ is the sum of any quantity $\bullet$ at two points separated by a distance $\vec{r}$. The quantities $\tilde{\epsilon}=\frac{\nu}{2} \overline{\left(\frac{\partial \tilde{u}_{i}}{\partial x_{j}}+\frac{\partial \tilde{u}_{j}}{\partial x_{i}}\right)^{2}}$ and $\epsilon^{\prime}=\frac{\nu}{2} \overline{\left(\frac{\partial u_{i}^{\prime}}{\partial x_{j}}+\frac{\partial u_{j}^{\prime}}{\partial x_{i}}\right)^{2}}$ are the mean energy dissipation rates of the coherent and the random motions, respectively.

For the sake of clarity, these equations are formally rewritten as

$$
\begin{aligned}
& \mathcal{I}_{c}+\mathcal{A}_{c m}+\mathcal{D}_{c c}+\mathcal{D}_{r c}^{\dagger}+\mathcal{D}_{c p}+\mathcal{P}_{c m}-\mathcal{P}_{r c}+\mathcal{T}_{c}+\mathcal{F}_{c}+\mathcal{V}_{c}=-2 \Sigma \overline{\tilde{\epsilon}} \\
& \mathcal{I}_{r}+\mathcal{A}_{r m}+\mathcal{D}_{r r}+\mathcal{D}_{r c}^{\dagger \dagger}+\mathcal{D}_{r p}+\mathcal{P}_{r m}+\mathcal{P}_{r c}+\mathcal{T}-\mathcal{T}_{c}-\mathcal{F}_{c}+\mathcal{V}_{r}=-2 \Sigma \overline{\epsilon^{\prime}}
\end{aligned}
$$

where $\mathcal{I}, \mathcal{A}, \mathcal{D}, \mathcal{P}, \mathcal{T}, \mathcal{F}$ et $\mathcal{V}$ denote respectively the non stationarity, advection, diffusion, production, transfer, forcing and viscous terms. The subscripts $m, c, r$ correspond to the mean, coherent and random motions, and $\mathcal{D}_{p}$ indicates the pressure diffusion. When two indices are present, the first one identifies the quantity on which that effect applies, whereas the second index points to the effect responsible for that effect (e.g., $\mathcal{D}_{r c}$ indicates the diffusion of RM through the CM). Further, we can shed light on $\mathcal{D}_{r c}^{\dagger}$ and $\mathcal{D}_{r c}^{\dagger \dagger}$, the transport of random statistical quantities by the organized motion.

By comparison with Danaila et al. (2002, 2004), there are additional terms which emerge in the present equations, e.g. the terms $\mathcal{P}_{r c}, \mathcal{T}_{c}$ and $\mathcal{F}_{c}$ which can be identified as the production of random fluctuations by the coherent motion, the coherent kinetic energy transfer and the forcing associated by the presence of a coherent motion. All three are present in equations (2.12) and (2.13), but with opposite signs. This means that the terms which can be identified as a loss of energy for the coherent motion (Eq. (2.12)), represent a gain of energy for randomly fluctuating motion (Eq. (2.13)). Equations (2.12) and (2.13) provide a general framework which allows the physics of the interaction between coherent and random fields to be unravelled.

In a globally homogeneous context, the limit at the largest scales of Eqs. (2.12) and 
(2.13) is twice the one-point energy budgets provided by Reynolds \& Hussain (1972)

$$
\begin{array}{r}
\frac{1}{2} \frac{D}{D t} \tilde{q}^{2}+\overline{\tilde{u}_{i} \tilde{u}_{j}} \frac{\partial \bar{U}_{i}}{\partial x_{j}}+\frac{\partial}{\partial x_{j}}\left(\overline{\tilde{u}_{j} \tilde{p}}+\frac{1}{2} \overline{\tilde{u}_{j} \tilde{q}^{2}}+\overline{\tilde{u}_{i}\left\langle u_{i}^{\prime} u_{j}^{\prime}\right\rangle}\right)-\overline{\left\langle u_{i}^{\prime} u_{j}^{\prime}\right\rangle \frac{\partial \tilde{u}_{i}}{\partial x_{j}}}=-\overline{\tilde{\epsilon}} \\
\frac{1}{2} \frac{D}{D t} q^{\prime 2}+\overline{u_{i}^{\prime} u_{j}^{\prime}} \frac{\partial \bar{U}_{i}}{\partial x_{j}}+\frac{\partial}{\partial x_{j}}\left(\overline{u_{j}^{\prime} p^{\prime}}+\frac{1}{2} \overline{u_{j}^{\prime} q^{\prime 2}}+\frac{1}{2} \overline{\tilde{u}_{j}\left\langle q^{\prime 2}\right\rangle}\right)+\overline{\left\langle u_{i}^{\prime} u_{j}^{\prime}\right\rangle \frac{\partial \tilde{u}_{i}}{\partial x_{j}}}=-\overline{\epsilon^{\prime}} .
\end{array}
$$

At this stage, Eqs. (2.12) and (2.13) are functions of the time $t$, the reference point vector $\vec{x}$ and the separation vector $\vec{r}$. This leads to a problem in 7 dimensions ( 8 before timeaveraging). In order to reduce the number of degrees of freedom, one generally invokes the local isotropy assumption. The practical consequence is that these isotropic forms lend themselves to be tested experimentally, with the constraint that two-point statistics are usually evaluated along one particular direction. Further, this allows us to compare the present considerations with those developed over the last half century (Kolmogorov 1941a; Yaglom 1949; Antonia et al. 1997; Danaila et al. 2002, 2004).

\subsubsection{Locally homogeneous and isotropic context}

First, if homogeneity holds at the level of the viscous scales, then the viscous term of e.g. the random motion reduces to (Hill 2001)

$$
-\nu\left[\left(2 \frac{\partial^{2}}{\partial r_{j}^{2}}+\frac{1}{2} \frac{\partial^{2}}{\partial X_{j}^{2}}\right) \overline{\Delta q^{\prime 2}}+2 \Sigma\left(\overline{\frac{\partial u_{i}^{\prime}}{\partial x_{j}} \frac{\partial u_{j}^{\prime}}{\partial x_{i}}}\right)\right]=-2 \nu \frac{\partial^{2}}{\partial r_{j}^{2}} \overline{\Delta q^{\prime 2}},
$$

Then, in a locally isotropic context, the divergence and the Laplacian operators are expressed as (Danaila et al. 2002, 2004)

$$
\frac{\partial}{\partial r_{j}}=\frac{2}{r}+\frac{\partial}{\partial r}, \quad \frac{\partial^{2}}{\partial r_{j}^{2}}=\left(\frac{2}{r}+\frac{\partial}{\partial r}\right) \frac{\partial}{\partial r} .
$$

By further using (2.17), after multiplying (2.12) and (2.13) by $r^{2}=r_{j} r_{j}$, integrating with respect to $r$ and dividing by $r^{2}$, we obtain

$$
\begin{array}{r}
\frac{1}{r^{2}} \int_{0}^{r} s^{2}\left(\mathcal{I}_{c}+\mathcal{A}_{c m}+\mathcal{D}_{c c}+\mathcal{D}_{r c}^{\dagger}+\mathcal{P}_{c m}-\mathcal{P}_{r c}+\mathcal{D}_{c p}\right) d s \\
+\overline{\Delta \tilde{u}_{\|} \Delta \tilde{q}^{2}}+\frac{2}{r^{2}} \int_{0}^{r} \overline{\Delta \tilde{u}_{i} \frac{\partial}{\partial s} s^{2}\left\langle\Delta u_{\|}^{\prime} \Delta u_{i}^{\prime}\right\rangle} d s-2 \nu \frac{\partial}{\partial r} \overline{\Delta \tilde{q}^{2}}=-\frac{4}{3} \overline{\tilde{\epsilon} r} \\
\frac{1}{r^{2}} \int_{0}^{r} s^{2}\left(\mathcal{I}_{r}+\mathcal{A}_{r m}+\mathcal{D}_{r r}+\mathcal{D}_{r c}^{\dagger \dagger}+\mathcal{P}_{r m}+\mathcal{P}_{r c}+\mathcal{D}_{r p}\right) d s \\
+\overline{\left\langle\Delta u_{\|} \Delta q^{2}\right\rangle}-\overline{\Delta \tilde{u}_{\|} \Delta \tilde{q}^{2}}-\frac{2}{r^{2}} \int_{0}^{r} \frac{\partial}{\Delta \tilde{u}_{i} \frac{\partial}{\partial s} s^{2}\left\langle\Delta u_{\|}^{\prime} \Delta u_{i}^{\prime}\right\rangle} d s-2 \nu \frac{\partial}{\partial r} \overline{\Delta q^{\prime 2}}=-\frac{4}{3} \overline{\epsilon^{\prime}} r .
\end{array}
$$

Eqs. (2.18a) and (2.18b) are the energy budget equations for the coherent and random components in a locally isotropic context. Here, $s$ is a dummy variable and the subscript II denotes the direction parallel to the separation vector. When the spatial separation is inferred using Taylor's hypothesis, this direction coincides with that of the mean flow.

The first line of Eqs. (2.18a) and (2.18b) represents the energy contribution of the largest scales (Danaila et al. 2002, 2004). The main difference with respect to the extended form of Kolmogorov equation (Antonia et al. 1997), is the appearance of several extra terms due to the presence of CM. The effective energy transfer of the random velocity component is explicit and consists of the total energy transfer $\overline{\left\langle\Delta u_{\|} \Delta q^{2}\right\rangle}$ (including the coherent and random contributions), from which are subtracted the coherent energy 


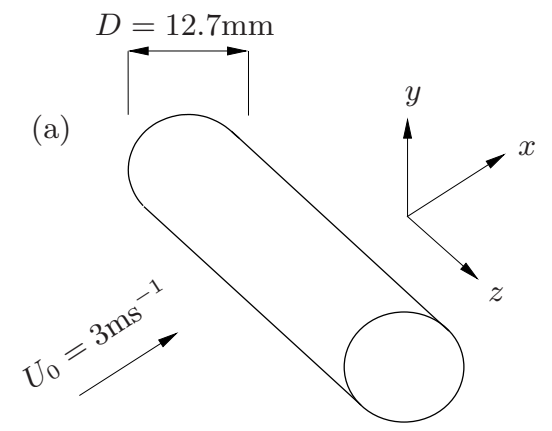

(b)

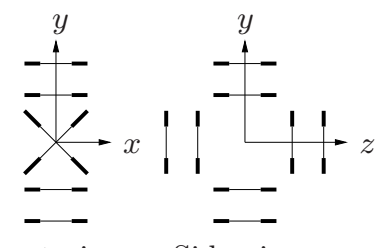

Front view Side view

FiguRE 1. (a) Definition of the coordinate system. (b) Sketches of the vorticity probe.

transfer $\overline{\Delta \tilde{u}_{\|} \Delta \tilde{q}^{2}}$ and the forcing term $\frac{2}{r^{2}} \int_{0}^{r} \overline{\Delta \tilde{u}_{i} \frac{\partial}{\partial s} s^{2}\left\langle\Delta u_{\|}^{\prime} \Delta u_{i}^{\prime}\right\rangle} d s$. In section 4, results based on experimental data will be shown.

\subsection{Experiments}

We recall briefly the most important features of the experimental set-up and measurement technique. The principal characteristics of the measurements reported here (performed at three downstream locations) are reported in Table 1. For more detailed information, the reader can refer to Zhou et al. (2003).

Measurements were carried out in an open-circuit wind tunnel with a working section of $0.35 \times 0.35 \mathrm{~m}^{2}$ and $2.4 \mathrm{~m}$ long. The cylinder of diameter $D=12.7 \mathrm{~mm}$ (Fig. 1 ) is placed horizontally, across the working section. The upstream velocity $U_{0}$ is $3 \mathrm{~ms}^{-1}$ corresponding to a Reynolds number $R e_{D}=\frac{U_{0} D}{\nu}=2540$ ( $\nu$ is the kinematic viscosity). Larger values of the upstream velocity $U_{0}$ than reported by Zhou et al. (2003), were also used, leading to larger values of the Taylor micro-scale Reynolds number $R_{\lambda}=\frac{\sqrt{\overline{u^{2}}} \lambda}{\nu} \cdot \overline{u^{2}}$ is the streamwise velocity variance, $\lambda$ is the Taylor micro-scale: $\lambda^{2}=15 \nu \overline{u^{2}} / \bar{\epsilon}$ and $\bar{\epsilon}$ the mean energy dissipation rate (see Zhou et al. (2003) for the values and the procedure employed for measuring $\bar{\epsilon}$ ). Measurements were made at different downstream locations: $x=10,20,40 D$, and different transverse positions, from $y=0$ to $y=3 D$.

A hot-wire probe, consisting of four cross-wires (Fig. 1) was used to measure simultaneously all three vorticity components (see Zhou et al. (2003) for more details about the probe).

Phase-averaged statistics are obtained as follows. The transverse velocity component $v$ was digitally band-pass filtered at the Strouhal frequency, using a eighth-order Butterworth filter. The filtering operation is implemented on the Fourier transform of $v$ in order to avoid any phase shift. As proposed by Perrin et al. (2007), the Hilbert transform $h$ of the filtered signal $v_{f}$ is subsequently calculated and the phase $\phi$ inferred from the relation $\phi=\arctan \left(\frac{h}{v_{f}}\right)$. Finally, the phase is divided into 41 segments and phaseaveraged statistics are calculated for each segment. The statistical convergence of calculated structure functions is known to be a common issue (Nichols-Pagel et al. 2008), and more especially when conditional structure functions are needed. In the present study, the convergence of statistics for second and third-order moments were checked, by reducing the number of segments for the phase, and found to be satisfactory. By means of our method, phase-averaged quantities are calculated over the period $[-\pi, \pi]$. As was done by O'Neil \& Meneveau (1997), the phase is doubled up to $[-2 \pi, 2 \pi]$ by taking advantage of the periodicity, in order to enhance the visual display.

In Marati et al. (2004), the geometrical space (location in the flow) and the separation 


$\begin{array}{rccc}x / D & 10 & 20 & 40 \\ \overline{u^{2}}\left(\mathrm{~m}^{2} \cdot \mathrm{s}^{-2}\right) & 0.276 & 0.133 & 0.045 \\ \overline{v^{2}}\left(\mathrm{~m}^{2} \cdot \mathrm{s}^{-2}\right) & 0.716 & 0.208 & 0.047 \\ \overline{w^{2}}\left(\mathrm{~m}^{2} \cdot \mathrm{s}^{-2}\right) & 0.137 & 0.097 & 0.42 \\ \bar{\epsilon}\left(\mathrm{m}^{2} \cdot \mathrm{s}^{-3}\right) & & & \\ L_{v} / D & 4.4 & 2.2 & 0.48 \\ \lambda(\mathrm{mm}) & 3.1 & 4.13 & 4.24 \\ \eta(\mathrm{mm}) & 0.15 & 0.70 & 0.29 \\ R_{\lambda} & 109 & 90 & 65\end{array}$

TABLE 1. Relevant flow parameters on the centerline of the wake for downstream distances of 10, 20 and 40D. The Reynolds number $R_{\lambda}=\left(\overline{u^{2}}\right)^{1 / 2} \lambda / \nu$, with $\lambda=\sqrt{15 \nu \overline{u^{2}} / \bar{\epsilon}}$ is based on the longitudinal velocity fluctuations since $u$ is weakly influenced by the CM.

space (turbulent scales) are made independent by considering the location specified by the midpoint $\vec{X}=\frac{1}{2}\left(\vec{x}+\overrightarrow{x^{+}}\right)$with $\overrightarrow{x^{+}}=\vec{x}+\vec{r}$. The same idea is applied here to phase-averaged structure functions for which the phase $\phi$ is defined as the phase at the midpoint $\phi=\phi(\vec{X})$. Defining $\phi=\phi(\vec{x})$ or $\phi=\phi\left(\overrightarrow{x^{+}}\right)$would have led to a linear dependence between $\phi$ and $r$.

\section{Results. Phase-averaged statistics in the wake flow}

\subsection{Flow topology and one-point, time-averaged quantities}

The kinetic energy of the organized motion is compared to that of the total fluctuating field (Figs. 2(a), 2(b), 2(c)). Ratios $\overline{\tilde{u}_{\alpha}^{2}} / \overline{u_{\alpha}^{2}}$ are calculated at several lateral $y / D$, as well as three downstream locations $(10 D, 20 D$ and $40 D)$.

On the wake centerline, one observes that the organized motion is mostly perceptible on the transverse velocity component $v$. Its energy contribution represents $\approx 70 \%$ of the transverse velocity variance at $10 D$, and is still perceptible at $40 D$ where $\overline{\widetilde{v}^{2}} / \overline{v^{2}} \approx 10 \%$. In contrast, $\overline{\tilde{u}^{2}}$ represents only $5 \%$ of the total variance at $x=10 D, y=0$ and is negligible beyond $20 D$. The location of the maximum of $\overline{\tilde{u}^{2}} / \overline{u^{2}}$ lies between $y=1 D$ and $y=2 D$, depending on the downstream distance. This transverse position is related to the position of the coherent structures which move away from the centerline when the downstream distance increases. At this particular transverse position, $\overline{\tilde{u}^{2}} / \overline{u^{2}} \approx \frac{1}{2} \overline{\tilde{v}^{2}} / \overline{v^{2}}$. Finally, the spanwise kinetic energy $w$ is entirely uncorrelated with the organized motion, as emphasized by Kiya \& Matsumura (1988) in the wake of a normal plate.

One of the main advantages of phase averaging is that it permits temporal and/or spatial dynamics associated with the presence of the organized motion to be emphasized. With regard to the wake flow, one generally displays statistics in the $(\phi, y)$ plane (Zhou \& Antonia 1995; Bisset et al. 1990) to relate e.g. the spatial organization of kinetic energy to that of the CM. The phase-averaged sectional streamlines of the halfwake are reported in Figs. 3(a), 3(b) and 3(c) for downstream positions varying from 10 to $40 \mathrm{D}$. These are calculated and displayed following the procedure of Bisset et al. (1990); Zhou \& Antonia (1993); Zhou et al. (2003). Sectional streamlines are observed in a frame of reference moving at a convection velocity of the coherent structures $U_{c}$ (see Zhou \& Antonia (1992); Lin \& Hsieh (2003); Zhou et al. (2003) for values of $U_{c}$ ), 


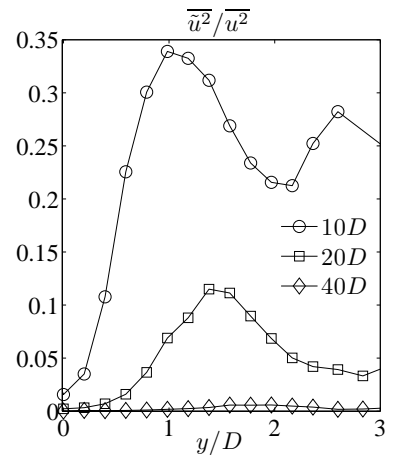

(a)

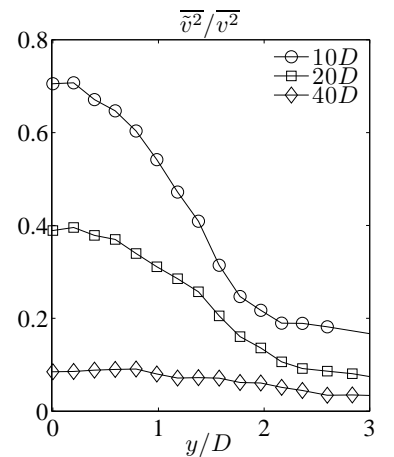

(b)

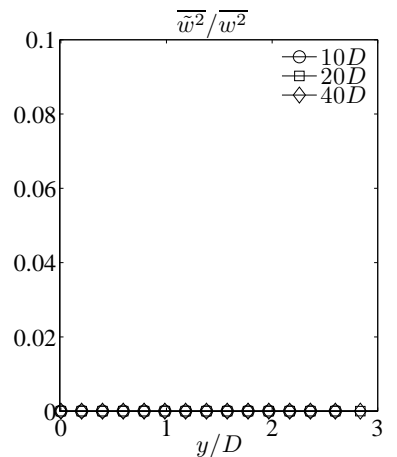

(c)

FIGURE 2. Energy contribution of the coherent motion. $\bigcirc: 10 D, \square: 20 D, \diamond: 40 D$. (a) $\overline{\widetilde{u}^{2}} / \overline{u^{2}}$, (b) $\overline{\widetilde{v}^{2}} / \overline{v^{2}}$, (c) $\overline{\widetilde{w}^{2}} / \overline{w^{2}}$.

the direction of which is from the right to the left, while the main flow is from left to right. Phase-averaged sectional streamlines reveal the position of the vortex cores which are centered around $\phi=\frac{\pi}{2} \pm 2 k \pi(k \in \mathbb{N})$ and at lateral positions varying between $0.25 D$ at $10 D$ and $1 D$ at $40 D$. The saddle points are located at $\phi=-\frac{\pi}{2} \pm 2 k \pi$ and $y \approx 1-1.5 D$ as $x$ increases. Moreover, near the cylinder the coherent structures are skewed along the $y$ direction, with the highest intensity located very nearly the wake centerline. Further downstream, the coherent structures cores are located away from the centerline $(y \approx 1 D)$, and they occupy a smaller volume due to the total circulation $(\Gamma)$ being very nearly conserved in the near-field of the wake.

We now turn our attention to the repartition of coherent kinetic energy in the $(\phi, y)$ plane. Iso-values of $\tilde{u}$ are reported in Figs. 3(d), 3(e), 3(f) and those of $\tilde{v}$ in Figs. 3(g), $3(\mathrm{~h}), 3(\mathrm{i})$. Note that $\phi=0 \pm k \pi$ and $y=0$ correspond to the location of the absolute maximum of the coherent transverse velocity fluctuation $\tilde{v}$. In contrast, local maxima of the longitudinal component $\tilde{u}$ is phase-shifted by $\phi=\frac{\pi}{2}$, with maximum values corresponding to the upper boundary of the positive vortex $\left(\phi=\frac{\pi}{2} \pm 2 k \pi\right)$ (Matsumura \& Antonia 1993). Minimum values are located at the upper boundary of vortices of negative sign $(\phi=\pi \pm 2 k \pi)$ (Matsumura \& Antonia 1993). Note also that on the wake centerline, $\tilde{u}$ reveals a periodicity of period $\pi$ due to the symmetric arrangement of vortices with respect to the centerline. In Figs. $3(\mathrm{j}), 3(\mathrm{k})$ and $3(\mathrm{l})$ is displayed the coherent strain $\widetilde{S}=\frac{\partial\langle U\rangle}{\partial y}+\frac{\partial\langle U\rangle}{\partial x}$ in the $(\phi, y)$ plane at the three downstream positions investigated. The coherent strain is of particular interest since it may induce an additional production term of both coherent and random fluctuations (Reynolds \& Hussain 1972). In the wake flow, this quantity reveals local maxima located at saddle points, i.e. $\phi=-\pi / 2 \pm 2 k \pi$. The strong connection between the energy distribution across turbulent scales and the coherent strain will be emphasized in subsection 3.2.

In summary, it is highlighted that the coherent motion is mostly discerned on $v$ with local extrema of $\tilde{v}$ located at $\phi=0 \pm k \pi$ and $y=0 d$. Phase averaged sectional streamlines show that coherent structures are centred around $\phi=\frac{\pi}{2} \pm 2 k \pi$ and $0.25 d<y<1 d$ depending on the streamwise location.

In the light of the previous remarks, this study will focus entirely on the wake centerline where the energy contribution of the coherent motion is maximum. In addition, this allows the effect of the coherent strain to be separated from that of the mean shear which is absent on the wake axis. It has been well established that the mean shear influences the 


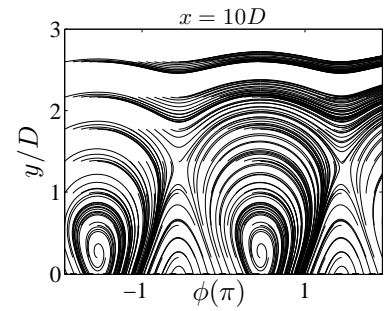

(a)

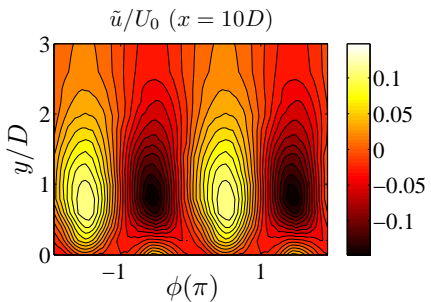

(d)

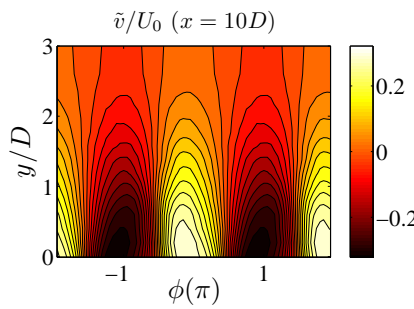

$(\mathrm{g})$

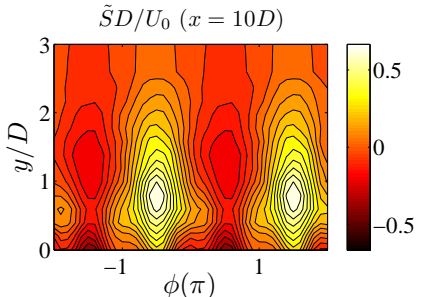

(j)

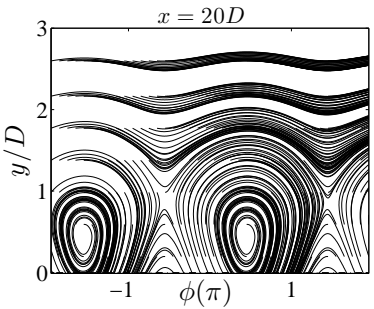

(b)

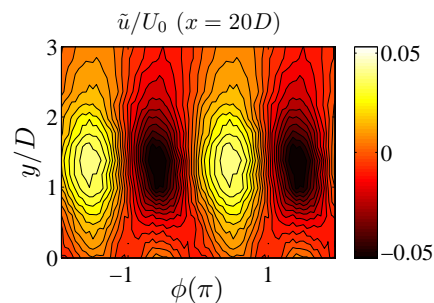

(e)

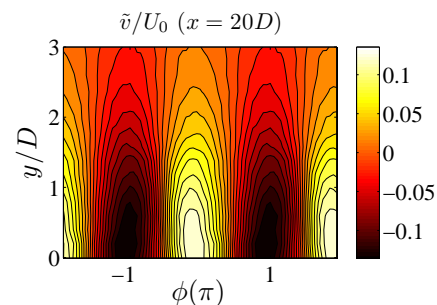

(h)

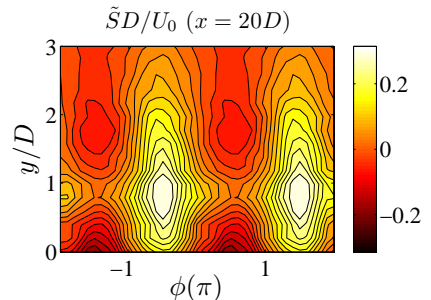

$(\mathrm{k})$

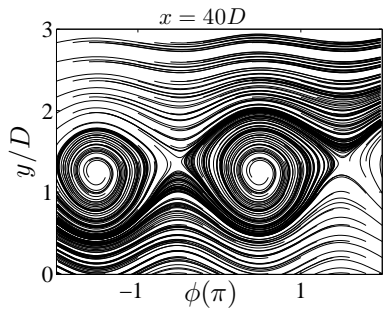

(c)

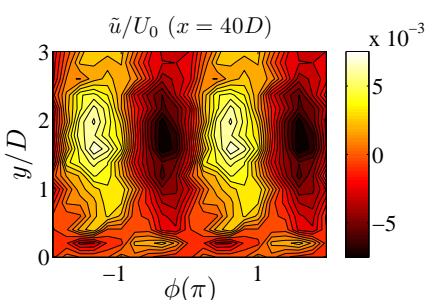

(f)

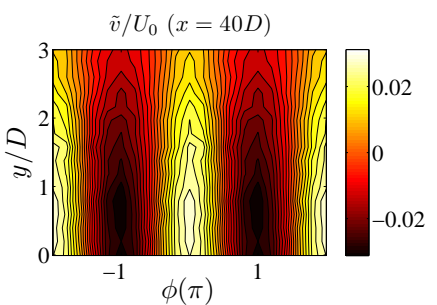

(i)

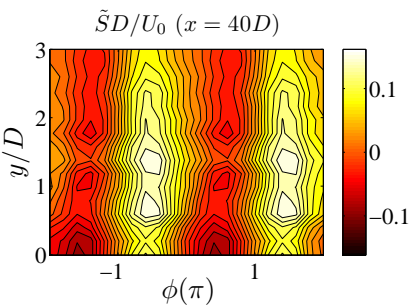

(1)

Figure 3. (a, b, c) Phase-averaged sectional streamlines. (d, e, f) Contours of $\widetilde{u} / U_{0}$. (g, h, i) Contours of $\widetilde{v} / U_{0} .(\mathrm{j}, \mathrm{k}, \mathrm{l})$ Contours of $\widetilde{S} D / U_{0}$. The flow direction is from left to right. Left, $x=10 D$; center, $x=20 D$; right, $x=40 D$.

behaviour of the smallest scales even at large Reynolds numbers (Praskovsky et al. 1993; Sreenivasan \& Dhruva 1998; Shen \& Warhaft 2000).

\subsection{Second-order phase-averaged structure functions}

We begin with phase-averaged second-order structure functions of the longitudinal velocity component $u$. We report the scale-by-scale energy distribution, of the total fluctuating field (Figs. 4(a), 4(b), 4(c)), of the coherent motion (Figs. 4(d), 4(e), 4(f)) and of the random part (Figs. $4(\mathrm{~g}), 4(\mathrm{~h}), 4(\mathrm{i}))$ in the $(\phi, r)$ plane, for $x$ varying from 10 to $40 D$. The separation $r$ is calculated by means of Taylor's hypothesis (i.e. $r=U_{c} \tau=U_{c} / F_{s}, F_{s}$ is the sampling frequency) and thus coincides with the direction of the mean flow $x$. The 


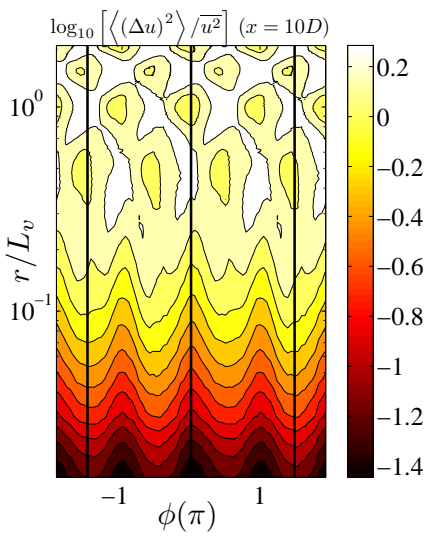

(a)

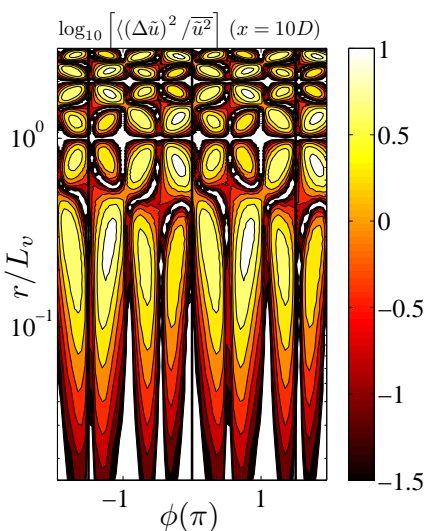

(d)

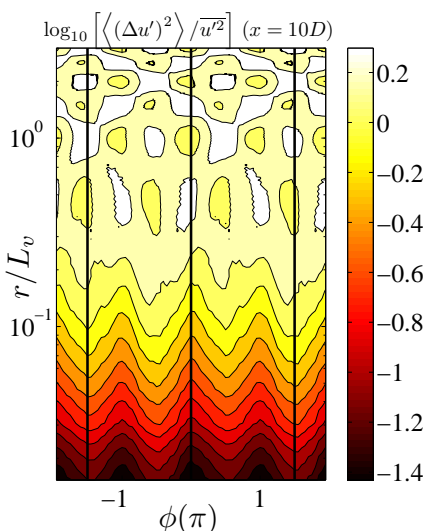

$(\mathrm{g})$

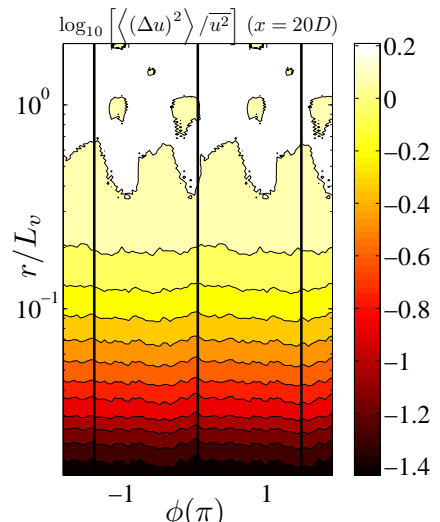

(b)

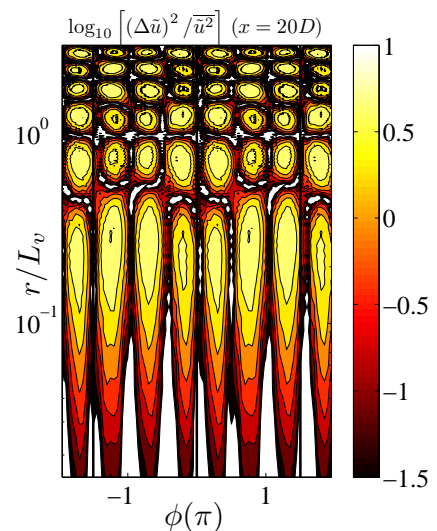

(e)

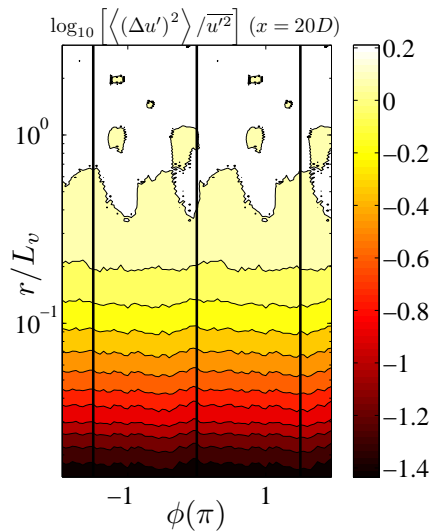

(h)

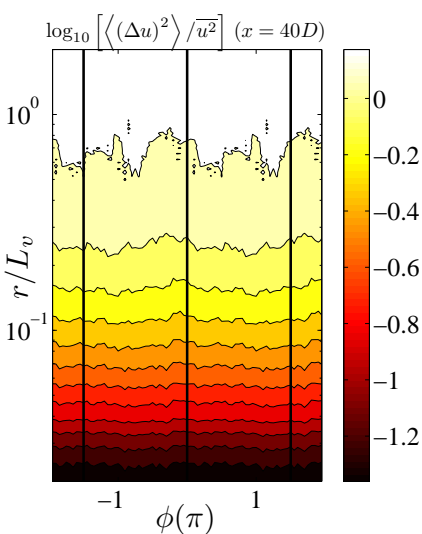

(c)

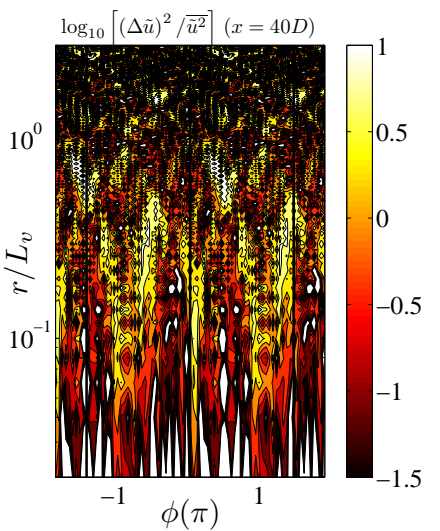

(f)

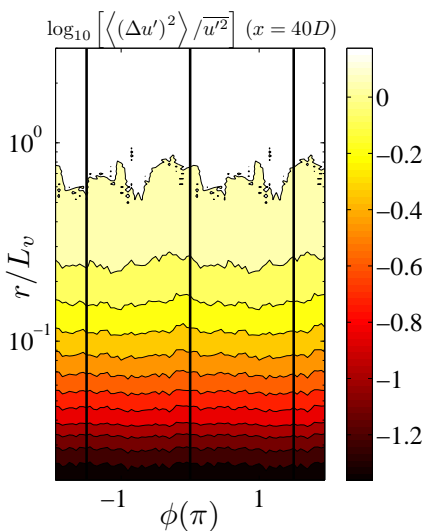

(i)

FiguRE 4. Phase-averaged second-order structure function of the longitudinal velocity component $u$ (in logarithmic scales $\left.\log _{10}\right)$. ( $\left.\mathrm{a}, \mathrm{b}, \mathrm{c}\right):\left\langle(\Delta u)^{2}\right\rangle / \overline{u^{2}}$. (d, e, f): $(\Delta \tilde{u})^{2} / \overline{\tilde{u}^{2}}$. (g, h, i): $\left\langle\left(\Delta u^{\prime}\right)^{2}\right\rangle \overline{u^{\prime 2}}$. Left $x=10 D$; center, $x=20 D$; right, $x=40 D$. Vertical lines correspond to $\phi=0$ and $\phi= \pm 3 \pi / 2$. 
$r$ axis is normalized by $L_{v}$, the streamwise distance between two consecutive vortices of the same sign; $L_{v}=4.15 D, 4.13 D$ and $4.24 D$ for $x=10,20$ and $40 D$ respectively.

A careful analysis of Figs. 4(a), 4(b), 4(c) reveals the range of scales which are influenced by the coherent motion. At $x=10 D$, the kinetic energy at the smallest scales is strongly enhanced at $\phi=-\frac{\pi}{2} \pm k \pi$. This phase location corresponds to the maxima (in amplitude) of the coherent strain. At 20D, the dependence of the phase is no longer visible at the smallest scales, but it still persists at the largest scales $\left(r / L_{v}>0.2\right)$. At $40 D$, the influence of the coherent motion among all values of the separation $r$ is not discernible.

Concerning the $(r, \phi)$-energy distribution of the coherent motion (Figs. 4(d), 4(e), 4(f)), we observe that $(\Delta \tilde{u})^{2}$ is zero for a phase $\phi=0 \pm k \frac{\pi}{2}$. Also, $(\Delta \tilde{u})^{2}=0$ for $r=\frac{L_{v}}{2}+k \frac{L_{v}}{2}$. This indicates that the periodicity in $u$ is half the distance between two vortices so that $\tilde{u}(x)-\tilde{u}\left(x+\frac{L_{v}}{2}+k \frac{L_{v}}{2}\right)=0$ and thus $\left\langle(\Delta u)^{2}\right\rangle\left(r=\frac{L_{v}}{2}+k \frac{L_{v}}{2}\right)=\left\langle\left(\Delta u^{\prime}\right)^{2}\right\rangle\left(\frac{L_{v}}{2}+k \frac{L_{v}}{2}\right)$. At $40 D$, the amplitude of $\tilde{u}$ is too small for the phase-average operation to reliably capture the energy distribution of the CM.

An important remark is that even though the $r$ axis is limited to $3 L_{v}$, the pattern observed in $(\Delta \tilde{u})^{2}$ continues indefinitely. Indeed, since the periodicity leads to a Dirac function in spectral space, the same periodicity has an infinite extent in physical space. Therefore, the localization in terms of scales of the coherent motion energy is lost in physical space, and its energy distribution is spread over an infinite range of scales.

The $(r, \phi)$ energy distribution of the random motion along the longitudinal direction, for downstream distances varying from $x=10 D$ to $40 D$, are plotted in Figs. 4(g), 4(h), 4(i). The topology of $\left\langle\left(\Delta u^{\prime}\right)^{2}\right\rangle(r, \phi)$ is very similar to that of the total fluctuating field. This reveals that the phase correlation observed in Figs. 4(a), 4(b) is not representative of the longitudinal coherent forcing $\tilde{u}$, whose topology is clearly different. In contrast, the phase correlation at small scales may be caused by a forcing in another direction, most likely in the $y$ direction associated with $\tilde{v}$. To unravel this issue, we now focus on the transverse velocity, whose contribution to the total kinetic energy is much more significant than that of $u$.

The phase-averaged transverse structure functions of the total fluctuating field $v$ (Figs. $5(\mathrm{a}), 5(\mathrm{~b}), 5(\mathrm{c})$ ), of the coherent component $\tilde{v}$ (Figs. $5(\mathrm{~d}), 5(\mathrm{e}), 5(\mathrm{f}))$, and of the randomly fluctuating field $v^{\prime}$ (Figs. $5(\mathrm{~g}), 5(\mathrm{~h}), 5(\mathrm{i})$ ) for downstream distances varying from 10 to $40 D$ are now presented. The phase-correlation of the total fluctuating field (Figs. 5(a), $5(\mathrm{~b}), 5(\mathrm{c})$ ) is clearly visible. A distance of $40 \mathrm{D}$ is needed before the phase-correlation disappears for small scales; however it is still perceptible for scales beyond $r=0.2 L_{v} \approx$ $2 \lambda$. As in the case of $u$, the energy at any scale $r$ of the transverse velocity component $v$ is enhanced at a phase $\phi=\frac{\pi}{2} \pm k \pi$ and is much smaller at a phase $\phi=0 \pm k \pi$. The topology of $\left\langle(\Delta v)^{2}\right\rangle$ is very analogous to that of $(\Delta \tilde{v})^{2}$ (Figs. 5(d), 5(e), 5(f)) which was not the case for $u$. For example, the phase $\pi$-periodicity and separation $L_{v^{-}}$ periodicity are recovered. Further, the local maxima and minima correspond to those of the coherent motion. Hence, this statistical tool allows us to assert that the coherent motion makes a strong energy contribution to the total fluctuating field, especially in the intermediate wake. By virtue of Eq. (2.6), we can calculate the random structure function of the velocity component $v$ (Figs. 5(g), 5(h), 5(i)). At 40D, the phase-averaged structure function is of similar shape to the usual time-averaged structure function, and the phase-correlation is lost. However, at $10 D$ and $20 D$, there is still a strong influence of the phase, even at very small scales. Note also that the topology in the $(r, \phi)$ plane is quite comparable to that of $u$, but amplitudes differ significantly (by a factor $\approx 2$ ).

We have emphasized that $\tilde{v}$ is the most important fluctuation. It is therefore important to dispose of a model for its statistics. Figures $5(\mathrm{~d}), 5(\mathrm{e}), 5(\mathrm{f})$ emphasize that the 


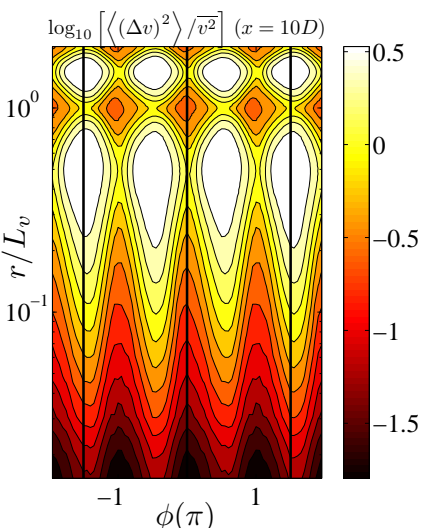

(a)

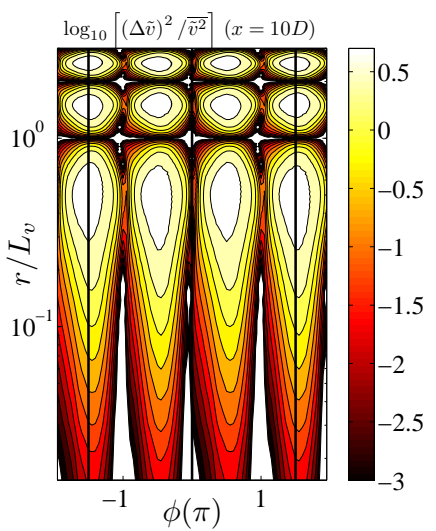

(d)

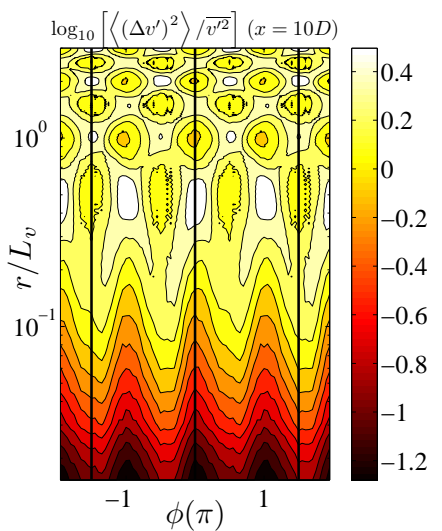

$(\mathrm{g})$

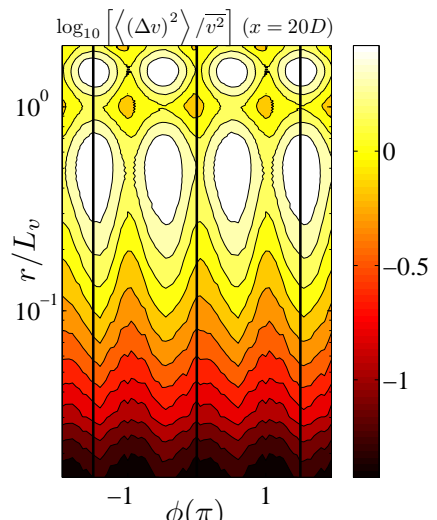

(b)

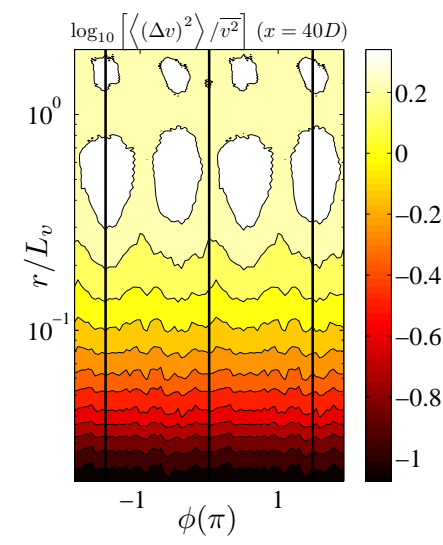

(c)

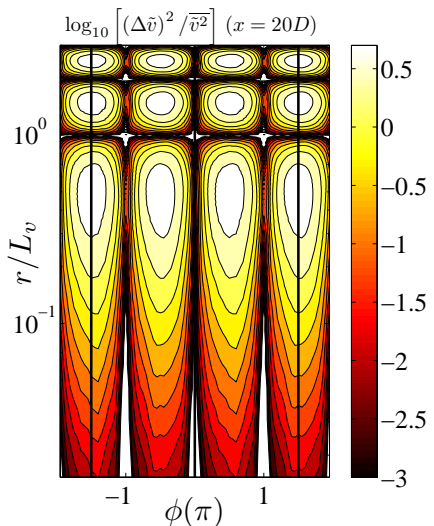

(e)

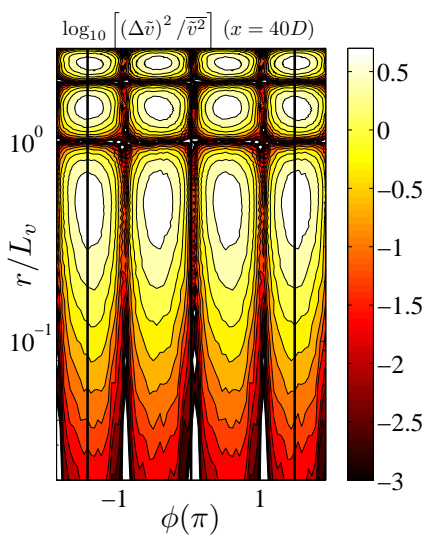

(f)

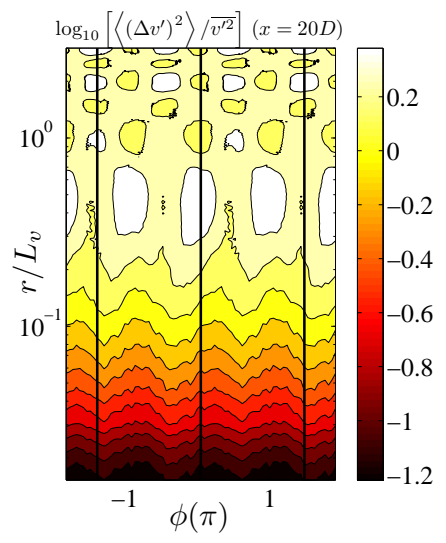

(h)

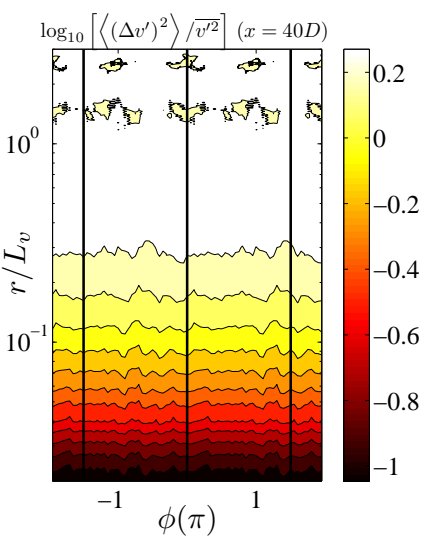

(i)

FiguRE 5. Phase-averaged second-order structure function of the transverse velocity component $v$ (in logarithmic scales $\left.\log _{10}\right)$. (a , b, c) $:\left\langle(\Delta v)^{2}\right\rangle \overline{v^{2}}$. (d, e, f): $(\Delta \tilde{v})^{2} / \overline{\tilde{v}^{2}} \cdot(\mathrm{g}, \mathrm{h}, \mathrm{i}):\left\langle\left(\Delta v^{\prime}\right)^{2}\right\rangle \overline{v^{\prime 2}}$. Left, $x=10 D$; center, $x=20 D$; right, $x=40 D$. Vertical lines correspond to $\phi=0$ and $\phi= \pm 3 \pi / 2$. 


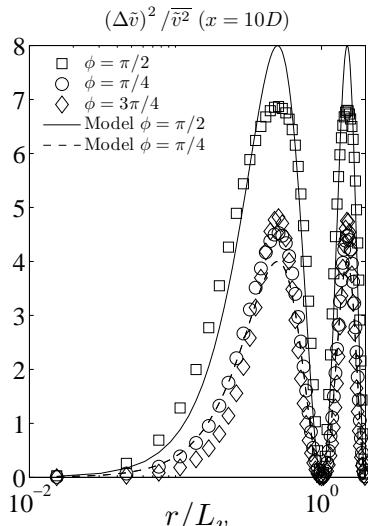

(a)

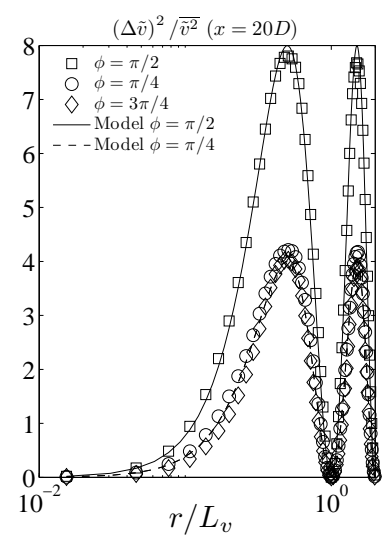

(b)

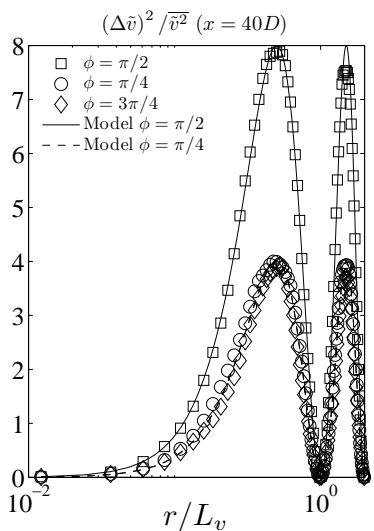

(c)

Figure 6. Phase-averaged second-order structure function of the coherent transverse velocity component $\tilde{v}:\left\langle(\Delta \tilde{v})^{2}\right\rangle \overline{\tilde{v}^{2}}$. Symbols $\square: \phi=\frac{\pi}{2}, \bigcirc: \phi=\frac{\pi}{4}, \diamond: \phi=\frac{3 \pi}{4}$. Comparison with the analytical expression Eq. (3.1). - - - : $\phi=\frac{\pi}{2}$. — : $\phi=\frac{\pi}{4}$ and $\phi=\frac{3 \pi}{4}$. (a) $10 D$, (b) $20 D$, (c) $40 D$.

coherent fluctuations contours in the $(\phi, r)$ plane are very similar. This particular feature indicates that a possible analytical expression can be applied to model the energy distribution among different scales. The phase-averaged structure function can be for example written as the product of two sinus functions parametrized by $L_{v}$, the streamwise distance between two consecutive vortices, and $\overline{\tilde{v}^{2}}$, the variance of the coherent part of the transverse velocity, viz.

$$
(\Delta \tilde{v})^{2} / \overline{\tilde{v}^{2}}=8 \sin ^{2}(\phi) \sin ^{2}\left(\pi \frac{r}{L_{v}}\right),
$$

Therefore, for $\phi=0 \pm k \pi,(\Delta \tilde{v})^{2}=0$ and for $\phi=\pi / 2 \pm k \pi(\Delta \tilde{v})^{2} / \overline{\tilde{v}^{2}}=8 \sin ^{2}\left(\pi \frac{r}{L_{v}}\right)$, as is observed experimentally. Further, $(\Delta \tilde{v})^{2}=0$ for $r=L_{v} \pm k L_{v}$, and $(\Delta \tilde{v})^{2} / \overline{\tilde{v}^{2}}=8 \sin ^{2}(\phi)$ for $r=L_{v} / 2 \pm k L_{v}$ in agreement with Figs. 5(d), 5(e), 5(f). Note also that, due to the symmetry of the sinus function

$$
(\Delta \tilde{v})^{2}\left(\phi \pm k \frac{\pi}{2}\right)=(\Delta \tilde{v})^{2}\left(-\phi \pm k \frac{\pi}{2}\right) .
$$

Then, the time-averaged structure function is obtained by integrating Eq. (3.1) over the period $\phi \in[-\pi ; \pi]$

$$
\overline{(\Delta \tilde{v})^{2}} / \overline{\tilde{v}^{2}}=4 \sin ^{2}\left(\pi \frac{r}{L_{v}}\right) .
$$

Equation (3.3) can be easily demonstrated by supposing $\tilde{v}=\cos \left(2 \pi \frac{x}{L_{v}}\right)$ and applying a spatial average over the period $[0 ; 2 \pi]$. In Figs. $6(\mathrm{a}), 6(\mathrm{~b}), 6(\mathrm{c})$, we compare the experimental phase-averaged structure functions with those modelled by Eq. (3.1) for the three values of $x / D$ and three different phase references. At $x=10 D$ (Fig. 6(a)), the model is qualitatively able to reproduce the shape of the phase-averaged structure functions. However, there are quantitative differences. For example, for $\phi=\frac{\pi}{2}$, the maximum value is weaker than that predicted by the model. In contrast, at the same phase reference, the model underestimates the energy distribution at the smallest scales. Further, the symmetry property Eq. (3.2) is violated since $(\Delta \tilde{v})^{2}\left(\frac{3 \pi}{4}\right) \neq(\Delta \tilde{v})^{2}\left(\frac{\pi}{4}\right)$. This asymmetry is 


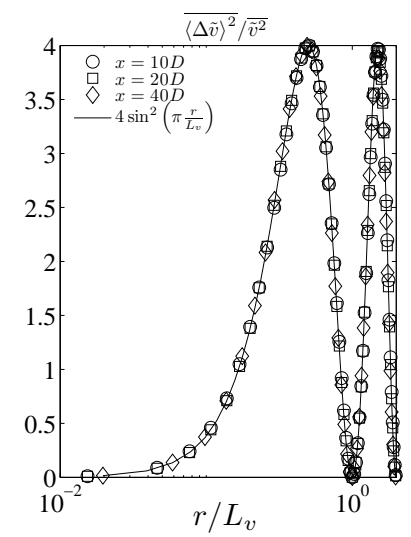

FIgURE 7 . Time-averaged second order structure function of the coherent velocity component v. —— Analytical expression based on Eq. (3.3), symbols $\bigcirc: 10 D, \square: 20 D, \diamond: 40 D$.

discernible in Fig. 5(d), and may be associated with another superposed sinusoidal signature. The latter may be phase-shifted from the main sinusoidal function that is accounted for in Eq. (3.1). At $x=20 D$ and $x=40 D$ (Fig. 6(b), 6(c)), the symmetry (Eq. (3.2)) is recovered and the sine-type model closely follows the experimental phase-averaged structure functions.

To summarize, the sine-type model for phase-averaged structure functions indicates only small departures from the measurements, especially at $10 D$. However, as emphasized by Fig. 7, the time-averaged structure function of the coherent motion is very well reproduced by Eq. (3.3). This provides very encouraging experimental support for the ability of this very simple model to represent the essential physics of the coherent motion, that is the distribution of energy among scales. In addition, let us recall that some analytical expressions of time-averaged structure functions have already been proposed, for example by Aivalis et al. (2002); Kurien \& Sreenivasan (2000), and recently successfully invoked by Antonia et al. (2003), Antonia \& Burattini (2006) in grid turbulence. These expressions which relate mostly to a purely random field can be added to Eq. (3.3) by virtue of Eq. (2.6) to assess the energy distribution of the total kinetic energy in a flow where a CM persists.

\subsection{Third-order phase-averaged structure functions}

We now turn our attention to third-order phase-averaged structure functions, and especially the mixed structure function $\left\langle\Delta u \Delta v^{2}\right\rangle$. After applying the triple decomposition to $\left\langle\Delta u \Delta v^{2}\right\rangle$ and with $\left\langle\Delta u_{\alpha}^{\prime}\right\rangle=0$ we obtain

$$
\left\langle\Delta u(\Delta v)^{2}\right\rangle=\Delta \tilde{u}(\Delta \tilde{v})^{2}+\left\langle\Delta u^{\prime}\left(\Delta v^{\prime}\right)^{2}\right\rangle+\Delta \tilde{u}\left\langle\left(\Delta v^{\prime}\right)^{2}\right\rangle+2 \Delta \tilde{v}\left\langle\Delta u^{\prime} \Delta v^{\prime}\right\rangle .
$$

Obviously, the phase-averaged third-order structure function of the random field can be assessed from Eq. (3.4), knowing $\Delta \tilde{u}$ and $\Delta \tilde{v},\left\langle(\Delta v)^{2}\right\rangle$ and $\left\langle\Delta u(\Delta v)^{2}\right\rangle$. In Figs. 8(a), 8(b) and 8(c), are represented the third-order structure functions of the total fluctuating field for $x=10, x=20 D$ and $x=40 D$ respectively. $\left\langle\Delta u \Delta v^{2}\right\rangle$ is divided by $\bar{\epsilon} r$, where $\bar{\epsilon}$ is the mean kinetic energy dissipation rate reported by Zhou et al. (2003).

First, one observes a strong small-scale phase-correlation, even at $40 \mathrm{D}$. This indicates that the phase-correlation is clearly much more significant for third-order moments than for second-order structure functions. We can thus expect that for higher moments and especially for fourth-order moments which give a first indication or measure of internal 


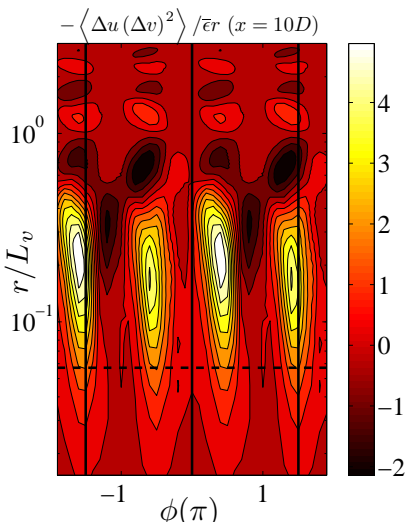

(a)

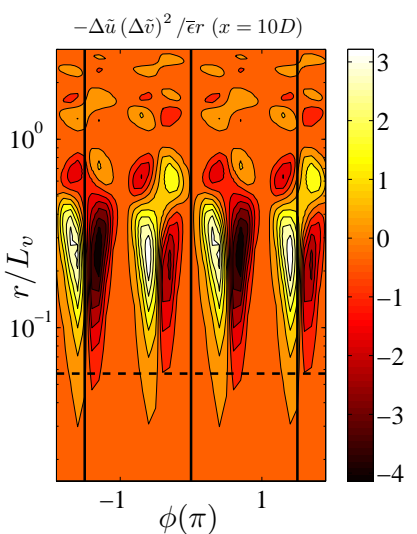

(d)

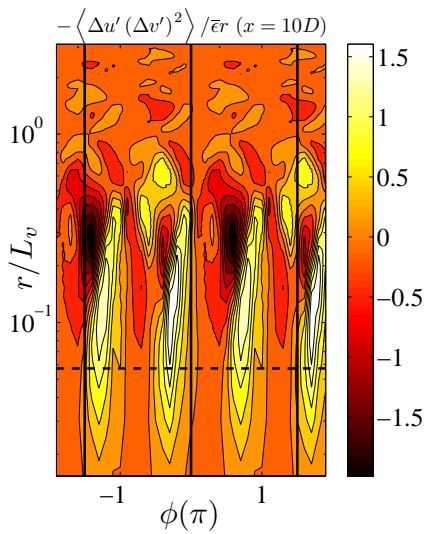

$(\mathrm{g})$

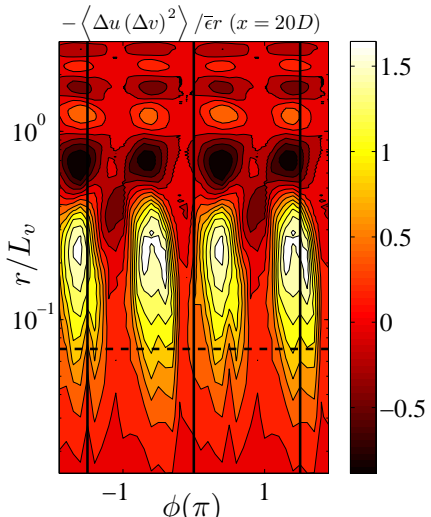

(b)

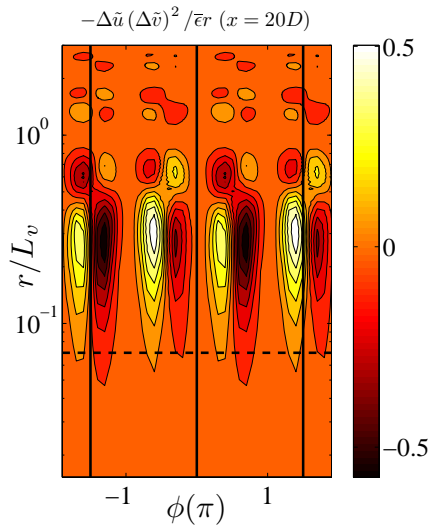

(e)

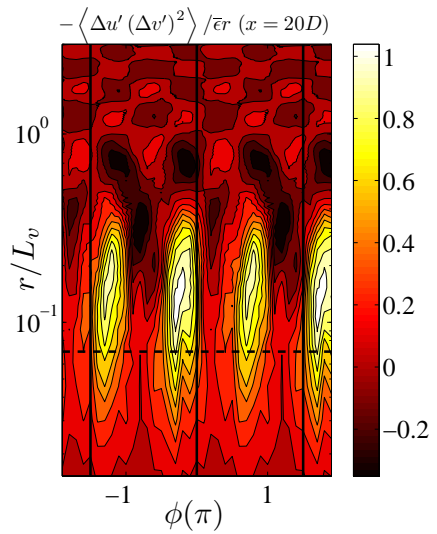

(h)

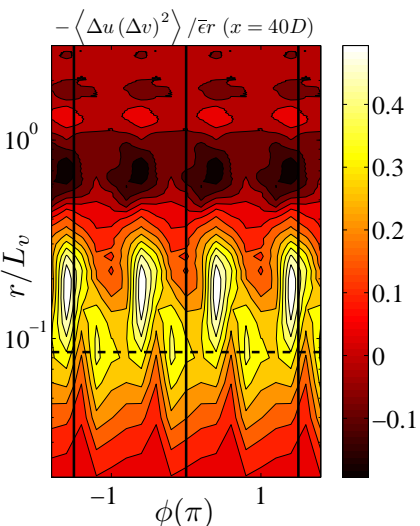

(c)

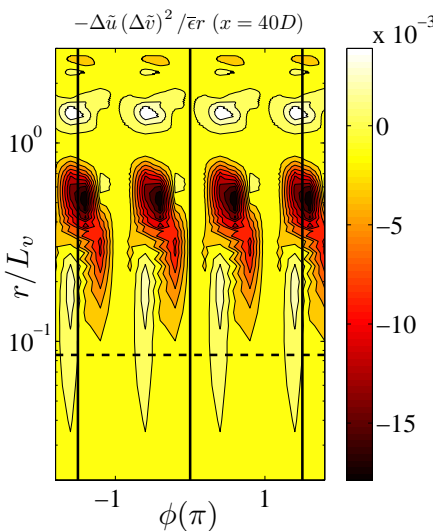

(f)

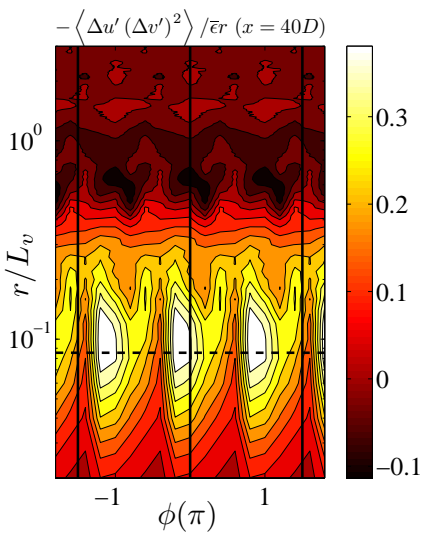

(i)

Figure 8. Phase-averaged third-order structure functions. (a , b, c): $-\left\langle\Delta u(\Delta v)^{2}\right\rangle / \bar{\epsilon}$. (d, e, f): $-\Delta \tilde{u}(\Delta \tilde{v})^{2} / \bar{\epsilon} r$. $(\mathrm{g}, \mathrm{h}, \mathrm{i}):-\left\langle\Delta u^{\prime}\left(\Delta v^{\prime}\right)^{2}\right\rangle / \bar{\epsilon} r$. left $x=10 D$, center $x=20 D$, right $x=40 D$. The horizontal dashed line represents the Taylor microscale $\lambda$. Vertical lines correspond to $\phi=0$ and $\phi= \pm 3 \pi / 2$. 
intermittency, the phase correlation may be more significant, whether the order of the moment is even or odd may also play a significant role. This issue is left for future work.

In the inertial range and in a locally isotropic context, we expect

$$
-\left\langle\Delta u(\Delta v)^{2}\right\rangle=-\frac{1}{3}\left\langle(\Delta u)^{3}\right\rangle \leq \frac{4}{15} \bar{\epsilon} r \approx 0.267 \bar{\epsilon} r .
$$

We observe that the maximum of the phase-averaged third-order structure functions can achieve some very high values, especially at $10 D$ and $20 D$ where $-\left\langle\Delta u(\Delta v)^{2}\right\rangle / \bar{\epsilon} r$ is $\approx 20$ and $\approx 6$ times greater than the expected isotropic value of $4 / 15$. These maxima are located at $\phi=\frac{\pi}{2} \pm k \pi$ where the coherent strain is maximum. This is the experimental evidence of a strong anisotropy of the total fluctuating field at rather small scales. The connection between the positions of the latter anisotropy and that of the coherent strain is fully consistent with the conclusions of Thiesset et al. (2013b). The coherent thirdorder structure function (Figs. 8(d), 8(e) and 8(f)), is not negligible at $10 D$ and tends to decrease as $x$ increases. At $x=40 D$, the maximum value of the third-order structure function is two orders of magnitude smaller than that of the total fluctuating field. However, irrespectively of $x$, since positive and negative coherent fluctuations are of the same amplitude, the time-averaged third-order structure function of the coherent motion is particularly weak.

In Fig. 8(g), 8(h) and 8(i), we represent the random phase-averaged third-order structure functions between $x=10 D$ and $x=40 D$. Here again, the phase correlation is easily discernible, and more accentuated than that of the second-order moments. At $x=20,40 D$, the maximum value exceeds the isotropic expectation of $4 / 15$, while at $40 D$ the maximum value is roughly consistent with isotropy. The phase location of these maxima is $\phi=\frac{\pi}{4} \pm k \pi$. By comparison with Fig. 8(a), 8(b) and 8(c), these maxima are also localized at smaller scales $(r \approx 1-2 \lambda)$ and have a smaller magnitude. Since the third-order structure function of the coherent motion is particularly small especially at $40 D$, the difference between $\left\langle\Delta u(\Delta v)^{2}\right\rangle$ and $\left\langle\Delta u^{\prime}\left(\Delta v^{\prime}\right)^{2}\right\rangle$ is mostly due to the other subtracted terms and particularly to $2 \Delta \tilde{v}\left\langle\Delta u^{\prime} \Delta v^{\prime}\right\rangle$ (not shown), which may be interpreted as the sweeping of the random Reynolds stress by the organized motion.

In summary, phase-averaged structure functions are an adequate tool for highlighting the influence of the coherent motion. The latter persists even at the smallest scales and relatively far downstream of where the energy was injected. The influence is more perceptible on third-order moments than on second-order moments. For example, at $x=$ $40 \mathrm{D}$, there is no more footprints of the coherent motion on phase-averaged second-order structure functions. However, the interaction persists in third-order moments. Other measurements were made with the same vorticity probe, at the same spatial locations, but at a higher Taylor microscale Reynolds number (up to $R_{\lambda} \approx 300$ ). Although these data are not reported in this study, they reveal that the scale beyond which the CM has an influence remains constant (in terms of $L_{v}$, which does not vary over the range of Reynolds numbers investigated). This observation was already predicted by Thiesset et al. (2013b) using dimensional arguments. Nevertheless, since the Taylor and Kolmogorov lengthscales progressively diminish as the Reynolds number increases, the range of scales which is not influenced by the dynamics of the CM extends. In other words, the degree of interactions between the CM and small scales must be understood as a finite Reynolds number effect and should be discernible especially at small Reynolds numbers. We can thus expect SSU to be recovered at infinite Reynolds numbers since the degree of the interactions between the CM and the small scale progressively diminishes.

On the other hand, we have to mention that it would be incorrect to conclude that the phase-correlation at the smallest scales is the experimental evidence for the existence of 
non-local interactions between separated scales. Indeed, it was shown that the coherent motion energy is not precisely localized in physical space (in contrast with the spectral space) and extends over an infinite range of scales. This conclusion queries the ability of second-order structure functions to describe effectively the energy at a particular scale r. According to Townsend (1956); Davidson \& Pearson (2005); Mouri \& Hori (2010); Danaila et al. (2012a), second-order structure functions represent the energy of every scale smaller than $r$ rather than the energy of a scale of typical size $r$.

\section{Experimental assessment of scale-by-scale energy budget}

At the centerline, the locally isotropic scale-by-scale budget of the random motion can be written

$$
\begin{array}{r}
-\frac{1}{r^{2}} \int_{0}^{r} s^{2} \mathcal{A}_{r m} d s-\overline{\left\langle\Delta u_{\|} \Delta q^{2}\right\rangle} \\
+\overline{\Delta \tilde{u}_{\|} \Delta \tilde{q}^{2}}+\frac{2}{r^{2}} \int_{0}^{r} \frac{\partial}{\Delta \tilde{u}_{i} \frac{\partial}{\partial s} s^{2}\left\langle\Delta u_{\|}^{\prime} \Delta u_{i}^{\prime}\right\rangle} d s+2 \nu \frac{\partial}{\partial r} \overline{\Delta q^{\prime 2}}=\frac{4}{3} \overline{\epsilon^{\prime}} r
\end{array}
$$

which means that in the limit of large scales, the advection term is almost entirely compensated by the energy dissipation rate; other large scale terms such as longitudinal production or turbulent diffusion are, as a first approximation, negligible compared to the advection term.

First, let us assess the adequacy of the local isotropy hypothesis on the wake centerline. To this end, we use the isotropic relation between second-order structure functions (Monin \& Yaglom 2007)

$$
\left.\overline{\left(\Delta u_{\perp}\right)^{2}}\right|_{i s o}=\overline{(\Delta u)^{2}}+\frac{r}{2} \frac{\partial}{\partial r} \overline{(\Delta u)^{2}}
$$

in which $u_{\perp}$ denotes either $v$ or $w$. In Fig. 9(a), we report the ratio between measured and calculating structure functions (using Eq. (4.2)) of the velocity component $v, v^{\prime}$ and $w$. If isotropy holds, the ratio must be equal to 1 for all values of $r$.

First, the spanwise velocity component $w$ closely follows the isotropic relation. The departure from local isotropy does not exceed $10 \%$. At the smallest scales, the transverse component $v$ respects isotropy and the ratio increases as we progress through to larger scales. The maximum deviation from isotropy is observed at $r / L_{v} \approx 0.5 \equiv r \approx 5 \lambda$ and the value of the ratio approaches $50 \%$. As was emphasized by Thiesset et al. $(2013 b)$, this departure from local isotropy can be explained by the presence of the coherent strain associated with the organized motion. However, statistics of the random part of the fluctuating transverse motion are much closer to the isotropic value, and Eq. (4.2) is respected with a maximum departure of about $30 \%$. Therefore, isotropy is roughly verified on the centerline of the wake for scales smaller than $\approx 3 \lambda$. For larger scales the departure from local isotropy attains $30 \%$ which does not seem unreasonable as far as experimental sources of errors are concerned.

Note that the local axisymmetry hypothesis should have been a more comfortable approximation since $\overline{\left(\Delta v^{\prime}\right)^{2}} \approx \overline{\left(\Delta w^{\prime}\right)^{2}}$ for $r<2-3 \lambda$. This indicates that a possible extension of the present work is to derive the axisymmetric energy budgets equations which account for the coherent motion, following the procedure outlined for example by Danaila et al. (2012b). However, the comparison with experiments is much more difficult since this requires the measurements of three-point correlation functions using, at least, two X-probes separated in the transverse direction (Sjögren \& Johansson 1998; Valente 


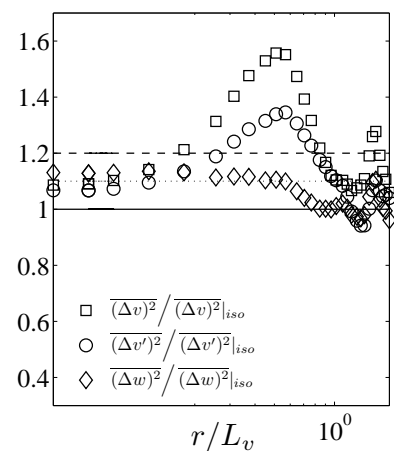

(a)

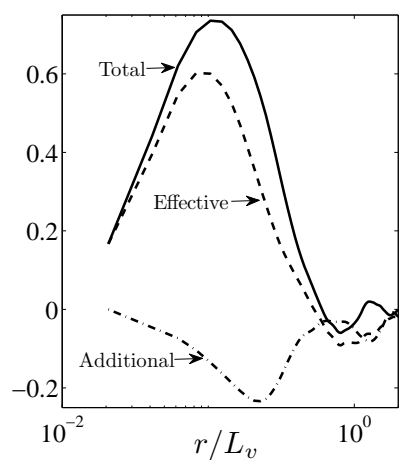

(b)

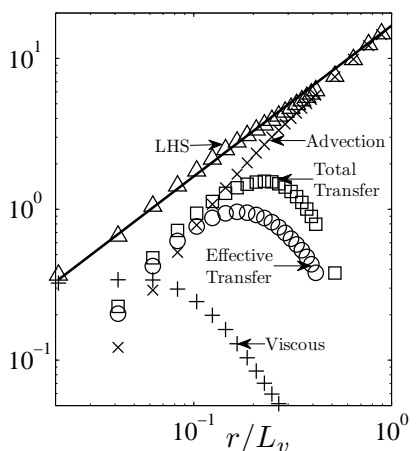

(c)

Figure 9. (a) Ratios between measured and calculated second-order structure functions. $\quad \square: \overline{(\Delta v)^{2}} /\left.\overline{(\Delta v)^{2}}\right|_{i s o}, \quad \bigcirc: \overline{\left(\Delta v^{\prime}\right)^{2}} /\left.\overline{\left(\Delta v^{\prime}\right)^{2}}\right|_{i s o}, \quad \diamond: \overline{(\Delta w)^{2}} /\left.\overline{(\Delta w)^{2}}\right|_{i s o} . \quad \cdots \cdots$ and - - - represent a departure of $10 \%$ and $20 \%$ respectively. (b) Non linear transfer term divided by $\overline{\epsilon^{\prime}} r$. — total transfer term $-\overline{\left\langle\Delta u_{\|} \Delta q^{2}\right\rangle}$, - - - coherent transfer and forcing term $\overline{\Delta \tilde{u}_{\|} \Delta \tilde{q}^{2}}+\frac{2}{r^{2}} \int_{0}^{r} \overline{\Delta \tilde{u}_{i} \frac{\partial}{\partial s} s^{2}\left\langle\Delta u_{\|}^{\prime} \Delta u_{i}^{\prime}\right\rangle} d s$, - - - Sum $-\overline{\left\langle\Delta u_{\|} \Delta q^{2}\right\rangle}+\overline{\Delta \tilde{u}_{\|} \Delta \tilde{q}^{2}}+\frac{2}{r^{2}} \int_{0}^{r} \overline{\Delta \tilde{u}_{i} \frac{\partial}{\partial s} s^{2}\left\langle\Delta u_{\|}^{\prime} \Delta u_{i}^{\prime}\right\rangle} d s$. (c) Scale-by-scale budget of the random motion (Eq. (4.1)) divided by $\overline{\epsilon^{\prime}}$. — : $\frac{4}{3} \overline{\epsilon^{\prime}} r, \times:-\frac{1}{r^{2}} \int_{0}^{r} s^{2} \mathcal{A}_{r m} d s, \square-\overline{\left\langle\Delta u_{\|} \Delta q^{2}\right\rangle}, \circ$ : $-\overline{\left\langle\Delta u_{\|} \Delta q^{2}\right\rangle}+\overline{\Delta \tilde{u}_{\|} \Delta \tilde{q}^{2}}+\frac{2}{r^{2}} \int_{0}^{r} \overline{\Delta \tilde{u}_{i} \frac{\partial}{\partial s} s^{2}\left\langle\Delta u_{\|}^{\prime} \Delta u_{i}^{\prime}\right\rangle} d s,+: 2 \nu \frac{\partial}{\partial r} \overline{\Delta q^{\prime 2}}, \Delta$ : left hand side of Eq. (4.1).

2013). For this reason, we are interested here only in the isotropic formulation of the scale-by-scale energy budgets.

Let us now turn our attention to the effective transfer term that was emphasized in the previous analytical section. Fig. 9(b) shows the total non linear transfer $-\overline{\left\langle\Delta u_{\|} \Delta q^{2}\right\rangle}$, the additional coherent transfer and forcing due to the coherent motion $\overline{\Delta \tilde{u}_{\|} \Delta \tilde{q}^{2}}+$ $\frac{2}{r^{2}} \int_{0}^{r} \overline{\Delta \tilde{u}_{i} \frac{\partial}{\partial s} s^{2}\left\langle\Delta u_{\|}^{\prime} \Delta u_{i}^{\prime}\right\rangle} d s$ as well as the effective transfer inferred from their sum, as a function of the separation $r / L_{v}$.

For weakly turbulent flows, the non linear transfer term is smaller than $\frac{4}{3} \bar{\epsilon} r$, because of the cross-over between viscous and large-scale effects (Danaila et al. 2002, 2004). Here, $-\overline{\left\langle\Delta u_{\|} \Delta q^{2}\right\rangle} / \overline{\epsilon^{\prime}} r \approx 0.63$. The additional energy transfer associated with the coherent motion is negative, its value being quite small, although not negligible. Its contribution is non zero for all separations with a maximum contribution at about $2 \lambda$. Finally, the maximum effective transfer of the random motion is smaller than the total transfer by about $12 \%$. Even though the difference of $12 \%$ between the total energy transfer and the effective energy transfer is not large, its influence may persist far from the injection of energy (Thiesset et al. 2013b) and thus remains the key for providing some insight into the energy transfer along the cascade in the wake flow, for which the CM is discernible. In a recent study (Thiesset et al. 2013a), it was observed that the maximum value of non linear transfer term measured in the wake of different generators was always larger than the one expected for grid turbulence for which the CM is absent. We thus prove here that this difference is associated with the presence of the CM whose macroscopic effect (i.e. when the RM and CM are not dissociated) is to enhance the total energy transfer.

The Reynolds number has also an influence on the amplitude of the effective energy 
transfer. Using the data at higher Reynolds numbers, it was observed that the difference between the total and the effective energy transfers diminishes as the Reynolds number is enhanced, and might be negligible beyond $R_{\lambda}=250-300$ for the circular cylinder wake. Therefore, the effect of the CM on the energy transfer must be interpreted as a finite Reynolds number effect. On the other hand, this difference between the total and the effective energy transfer depends also on initial conditions since different generators lead to different amplitudes of the coherent motion (see e.g. Antonia et al. (2002); Thiesset et al. $(2013 b, a))$. Therefore, the Reynolds number that is required for the forcing due to the $\mathrm{CM}$ to be negligible depends on initial conditions, and is expected to be larger for porous generators than for impervious ones. The extra-transfer term is non zero over a large range of scales. However, one cannot claim that this quantifies the non local interactions between coherent and random fields because of the loss of localization in physical space, as mentioned previously.

The balance between right and left sides of (4.1) is reasonably satisfied at all scales (Fig. 9(c)). The weak imbalance at rather large scales implies that either local isotropy no longer holds or that other production and/or diffusion terms must be taken into account in Eq. (4.1).

\section{Conclusions}

The connection between the temporal dynamics of the coherent motion and the energy distribution across all scales has been highlighted by means of phase-averaged structure functions. This original statistical tool allows us to assess the range of scales affected by the CM dynamics. Moreover, we are able to separate the energy contributions of the coherent and random fluctuations. Phase-averaging the structure functions measured in a cylinder wake yields three main outcomes.

First, it is shown that, as the distance downstream of the cylinder increases, (i) the scale at which the forcing associated with the CM is perceptible continually increases, (ii) phase-scale structure functions reveal also that a scale $r$ is correlated with that of the coherent strain, the effect of the latter being to locally enhance the energy at any scale $r$.

Second, we have proposed a simple analytical expression for the CM energy distribution. This treatment relies on two parameters that may depend on the type flow: the length scale $L_{v}$ and the variance $\overline{\tilde{v}^{2}}$. This expression can be used together with available analytical expressions for the second-order structure functions which relate to the random motion, therefore providing an analytical description of the total fluctuating field statistics.

Finally, energy budget equations which account for the organized motion are derived. Both general and isotropic formulations are obtained. They highlight a few additional terms. One of these is interpreted as an additional forcing exerted by the CM on the random motion. At $x=40 D$, this term represents about $12 \%$ of the total transfer term, but its influence may persist far downstream. The isotropic formulation is tested against experimental data at the wake centerline. The weak imbalance between the analytical formulation and the measurements at rather large scales appears to underline the inadequacy of local isotropy at these scales.

\section{Acknowledgements}

The financial support of the 'Agence Nationale de la Recherche' (ANR), under the projects 'ANISO' and 'MUVAR', is gratefully acknowledged. RAA acknowledges the 
support of the Australian Research Council. FT is grateful for the hospitality of the CORIA laboratory during his $\mathrm{PhD}$, where the results presented here were first examined. He especially thanks G. Godard for his very useful help and the region Haute-Normandie for the financial support. We thank T. Zhou who carried out the original measurements at the University of Newcastle.

\section{REFERENCES}

Aivalis, K. G., Sreenivasan, K. R., Tsuji, Y., Klewicki, J.C. \& Biltoft, C. A. 2002 Temperature structure functions for air flow over moderately heated ground. Phys. Fluids 14, 2439-2446.

Antonia, R. A. \& Burattini, P. 2006 Approach to the 4/5 law in homogeneous isotropic turbulence. J. Fluid Mech. 550, 175-184.

Antonia, R. A., Ould-Rouis, M., Anselmet, F. \& Zhu, Y. 1997 Analogy between predictions of Kolmogorov and Yaglom. J. Fluid Mech. 332, 395-409.

Antonia, R. A., Smalley, R. J., Zhou, T., Anselmet, F. \& Danaila, L. 2003 Similarity of energy structure functions in decaying homogeneous isotropic turbulence. J. Fluid Mech. 487, 245-269.

Antonia, R. A., Zhou, T. \& Romano, G. P. 2002 Small-scale turbulence characteristics of two-dimensional bluff body wakes. J. Fluid Mech. 459, 67-92.

Bisset, D. K., Antonia, R. A. \& Browne, L. W. B. 1990 Spatial organization of large structures in the turbulent far wake of a cylinder. J. Fluid Mech. 218, 439-461.

Blum, D. B., Bewley, G. P., Bodenschatz, E., Gibert, M., Gylfason, A., Mydlarski, L., Voth, G. A., Xu, H. \& Yeung, P. K. 2011 Signatures of non-universal large scales in conditional structure functions from various turbulent flows. New J. Phys. 13, 113020.

Blum, D. B., Kunwar, S. B., Johnson, J. \& Voth, G. A. 2010 Effects of nonuniversal large scales on conditional structure functions in turbulence. Phys. Fluids 22, 015107.

Brown, G.L. \& Roshko, A. 1974 On density effects and large structure in turbulent mixing layers. J. Fluid Mech. 64, 775.

Brown, G. \& Roshko, A. 2012 Turbulent shear layers and wakes. J. Turbulence 13, 1-32.

Cambon, C, Danaila, L, Godeferd, FS \& Scott, JF 2013 Third-order statistics and the dynamics of strongly anisotropic turbulent flows. Journal of Turbulence 14 (3), 121-160.

Cambon, Claude \& Gréa, B-J 2013 The role of directionality on the structure and dynamics of strongly anisotropic turbulent flows. Journal of Turbulence 14 (1), 50-71.

Danaila, L., Anselmet, F. \& Antonia, R. A. 2002 An overview of the effect of large scale inhomogeneities on small-scale turbulence. Phys. Fluids 14, 2475-2484.

Danaila, L., Anselmet, F. \& Zhou, T. 2004 Turbulent energy scale-budget equations for nearly homogeneous sheared turbulence. Flow, Turbulence and Combustion 72, 287-310.

Danaila, L., Antonia, R. A. \& Burattini, P. $2012 a$ Comparison between kinetic energy and passive scalar energy transfer in locally homogeneous isotropic turbulence. Phys. D 241, $224-231$.

Danaila, L., Krawczynski, J.F., Thiesset, F. \& Renou, B $2012 b$ Yaglom-like equation in axisymmetric anisotropic context. Phys. D 241, 216223.

Davidson, P. A. \& Pearson, B. R. 2005 Identifying turbulent energy distributions in real, rather than fourier, space. Phys. Rev. Lett. 95, 214501.

Domaradzki, J Andrzej, Metcalfe, Ralph W, Rogallo, Robert S \& Riley, James J 1987 Analysis of subgrid-scale eddy viscosity with use of results from direct numerical simulations. Phys. Rev. Lett. 58 (6), 547.

HiLl, R. J. 2001 Equations relating structure functions of all orders. J. Fluid Mech. 434, 379388.

Hosokawa, I. 2007 A paradox concerning the refined similarity hypothesis of Kolmogorov for isotropic turbulence. Prog. Theor. Phys. 118, 169.

Kang, H. S. \& Meneveau, C. 2002 Universality of large eddy simulation model parameters across a turbulent wake behind a he ated cylinder. J. Turb. 3, 1-27.

Kholmyansky, M. \& Tsinober, A. 2008 Kolmogorov 4/5 law, nonlocality, and sweeping decorrelation hypothesis. Phys. Fluids 20, 041704. 
Kiya, M. \& Matsumura, M. 1988 Incoherent turbulence structure in the near wake of a normal plate. J. Fluid Mech. 190, 343-356.

Kolmogorov, A. 1941 a Dissipation of energy in the locally isotropic turbulence. Dokl. Akad. Nauk. SSSR 125, 15-17.

Kolmogorov, A. $1941 b$ The local structure of turbulence in incompressible viscous fluid for very large Reynolds numbers. Proc. USSR Ac. of Sci. 30, 299-303.

Kurien, S. \& Sreenivasan, K. R. 2000 Anisotropic scaling contributions to high-order structure functions in high-Reynolds-number turbulence. Phys. Rev. E 62, 2206-2212.

Lin, C. \& Hsien, S. C. 2003 Convection velocity of vortex structures in the near wake of a circular cylinder. J. Engrg Mech. 129, 1108-1118.

Marati, N., Casciola, C. M. \& Piva, R. 2004 Energy cascade and spatial fluxes in wall turbulence. J. Fluid Mech. 521, 191-215.

Matsumura, M. \& Antonia, R. A. 1993 Momentum and heat transport in the turbulent intermediate wake of a circular cylinder. J. Fluid Mech. 250, 651-668.

Monin, A. S. \& Yaglom, A. M. 2007 Statistical Fluid dynamics, , vol. 2. John L. Lumley.

Mouri, H. \& HorI, A. 2010 Two-point velocity average of turbulence: Statistics and their implications. Phys. Fluids 22, 115110.

Nichols-Pagel, Gerald A, Percival, Donald B, Reinhall, Per G \& Riley, James J 2008 Should structure functions be used to estimate power laws in turbulence? a comparative study. Phys. D 237 (5), 665-677.

O’Neil, J. \& Meneveau, C. 1997 Subgrid-scale stresses and their modelling in a turbulent plane wake. J. Fluid Mech. 349, 253-293.

Perrin, R., Braza, M., Cid, E., Cazin, S., Barthet, A., Sevrain, A., Mockett, C \& Thiele, F. 2007 Obtaining phase averaged turbulence properties in the near wake of a circular cylinder at high reynolds number using POD. Exp. Fluids 43, 341-355.

Praskovsky, A. A., Gledzer, E., Karyakin, M. Y. \& Zhou, Y. 1993 The sweeping decorrelation hypothesis and energy-inertial range interaction in high Reynolds number flow. $J$. Fluid Mech. 248, 493-511.

Reynolds, W. C. \& Hussain, A. K. M. F. 1972 The mechanics of an organised wave in turbulent shear flow. Part 3. Theoretical models and comparisons with experiments. J. Fluid Mech. 54, 263-288.

Roshko, A. 1954 On the development of turbulent wakes from vortex streets. Tech. Rep.. NACA TR.

Shen, X. \& WARhaft, Z. 2000 The anisotropy of the small scale structure in high Reynolds number $\left(\mathrm{R}_{\lambda}=1000\right)$ turbulent shear flow. Phys. Fluids 12, 2976-2989.

SJögren, T. \& Johansson, A. V. 1998 Measurement and modelling of homogeneous axisymmetric turbulence. J. Fluid Mech. 374, 59-90.

Sreenivasan, K. R. \& Antonia, R. A. 1997 The phenomenology of small-scale turbulence. Annu. Rev. Fluid Mech. 29, 435-472.

Sreenivasan, K. R. \& Dhruva, B. 1998 Is there a scaling in high Reynolds-number turbulence ? Prog. Theor. Phys. 130, 103-120.

Tchoufag, J., Sagaut, P. \& Cambon, C. 2012 Spectral approach to finite reynolds number effects on kolmogorovs 4/5 law in isotropic turbulence. Phys. Fluids 24, 015107.

Tennekes, H. 1975 Eulerian and Lagrangian time microscales in isotropic turbulence. J. Fluid Mech. 67, 561-567.

Thiesset, F., Antonia, R. A. \& Danaila, L. 2013a Scale-by-scale turbulent energy budget in the intermediate wake of two-dimensional generators. Phys. Fluids 25 (11), 115105.

Thiesset, F., Danaila, L. \& Antonia, R. A. $2013 b$ Dynamical effect of the total strain induced by the coherent motion on local isotropy in a wake. J. Fluid Mech. 720, 393-423.

Thiesset, F., Danaila, L., Antonia, R. A. \& Zhou, T. 2011 Scale-by-scale energy budgets which account for the coherent motion. J. Phys. Conference series 318, 052040.

Townsend, A. A. 1956 The structure of turbulent shear flows. Cambridge University Press.

VALENTE, P. 2013 Energy transfer and dissipation in equilibrium and nonequilibrium turbulence. $\mathrm{PhD}$ thesis, Imperial College.

Williamson, C.H.K. 1996 Vortex dynamics in the cylinder wake. Annu. Rev. Fluid. Mech. 28, 477-539. 
YAGLOM, A. M 1949 On the local structure of a temperature field in a turbulent flow. Dokl. Akad. Nauk SSSR 69, 743.

Zhou, T., Zhou, Y., Yiu, M.W \& ChuA, L.P 2003 Three-dimensional vorticity in a turbulent cylinder wake. Exp. Fluids 35, 459-471.

Zhou, Y. \& Antonia, R. A. 1992 Convection velocity measurements in a cylinder wake. Exp. Fluids 13, 63-70.

Zhou, Y. \& Antonia, R. A. 1993 A study of turbulent vortices in the near wake of a cylinder. J. Fluid Mech. 253, 643-661.

Zhou, Y. \& Antonia, R. A. 1995 Memory effects in a turbulent plane wake. Exp. Fluids 19, $112-120$. 